ARGONNE NATIONAL LABORATORY 9700 South Cass Avenue

Argonne, Illinois 60439
ANL/MCS-TM-172

DE93 004735

ANL/MCS-TM-172

An Entry in the 1992 Overbeek Theorem-Proving Contest

by

Ewing L. Lusk and William W. McCune

Mathematics and Computer Science Division

Technical Memorandum No. 172

November 1992

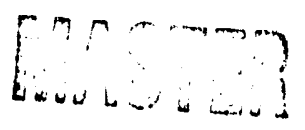

This work was supported by the Applied Mathematical Sciences subprogram of the Office of Energy Rescarch, U.S. Department of Energy, under Contract W-31-109-Eng-38. 


\section{Contents}

$\begin{array}{ll}\text { Abstract } & 1\end{array}$

1 Introduction 1

2 Results 1

3 Settings and Set of Support 2

3.1 Settings for the Basic Set $\ldots \ldots \ldots \ldots \ldots$

3.2 Settings for the Equality Set . . . . . . . . . . . . . 2

3.3 Description of the Settings . . . . . . . . . . . . . 2

4 Failures on Equality Theorems 6-10 5

5 Summary of OTTER Outputs for the Basic Set 6

5.1 Theorem 1: $x^{2}=e$ Groups are Commutative $($ P-form $) \ldots \ldots \ldots$

5.2 Theorem 2: The Commutator Theorem (P-form) . . . . . . . . 8

5.3 Theorem 3. $x^{2}=x$ Rings are Commutative $($ P-form $) \ldots \ldots \ldots 11$

5.4 Theorem 4: Equivalential Calculus, XGK $\rightarrow$ PYO . . . . . . . 14

.5.5 Theorem 5: Implicational Calculus Single Axiom, CD-67 (Imp-4) . . . . 16

5.6 Theorem 6: Many-valued Sentential Calculus, CD-57 . . . . . . . . . 19

5.7 Theorem 7 : Many-valued Sentential Calculus, CD-60 . . . . . . . 21

6 Summary of OTTER Outputs for the Equality Set 23

6.1 Theorem EQ-1: The Commutator Theorem . . . . . . . . . . . 23

6.2 Theorem EQ-2: Robbins Algebra, $(\exists c, c+c=c) \rightarrow$ Boolean . . . . . . 25

6.3 Theorem EQ-3: On Ternary Boolean Algebra . . . . . . . . . . . 28

6.4 Theorem EQ-4: Group Theory Single Axiom . . . . . . . . . . . 31

6.5 Theorem EQ-5: On Wajsberg Algebra . . . . . . . . . . . 35

$\begin{array}{lll}7 & \text { Conclusion } & 38\end{array}$

$\begin{array}{ll}\text { References } & 39\end{array}$ 


\title{
An Entry in the 1992 Overbeek Theorem-Proving Contest
}

\author{
by \\ Euing L. Lusk and William W. McCune
}

\begin{abstract}
A.bstract
At ('ADE-10 Ross Overbeek proposed a contest to stimulate and reward work in automated theorem proving. This paper represents an entry, or perhaps a family of related entries, in the contest.
\end{abstract}

\section{Introduction}

The ('onference on Automated Deduction (CADE) has been for nearly twenty years a menting where both theoreticians and system implementors present their work. Feeling perhaps that the conference was becoming dominated by the theoreticians, Ross Overbeek proposed at CADE-10 in 1990 a contest to stimulate work on the implementation and use of theorem-proving systems. The challenge was to prove a set of theorems, and do so with a uniform approach. That is, it was not allowed to set parameters in the system to specialize it for individual problems. There were actually two separate contests, one represented by a set of seven problems designed to test basic inference components, and the other represented by a set of ten problems designed to test equality-based systems.

This paper describes our experiences in preparing to enter the contest with OTTER $[5,6]$ and Roo [1, 2], two systems developed at Argonne National Laboratory. Roo is a parallel version of OTTER, but has such different behavior in some cases that we treat them as scparate entries. Wo entered each of them in both contests.

Some of the problems are difficult ones; and although many of the problems had been done before with OTTER, in each case we had set OTTER's many input parameters in a way customized to the problem at hand, and chosen a set of support that appeared to us to be most natural. It was a challenge to come up with a uniform set of parameter settings and a uniform algorithm for picking the set of support that would allow OTTER to prove each of the theorems.

\section{Results}

OTTER and ROO proved all seven theorems in the basic set first five of the ten prc ilems in the equality set. See Section 3 for the options settings and set of support used.

Tahles 1 and 2 list the results on the two sets for OTTER, for Roo running with 8 processors, and for Roo with 12 processors. 
The OTTER jobs were run on SPAR(station?. We used OTTER 2.2, the version that was relrased in July 1991. The Roo jobs were run on an Alliant 2800 with 12 (Intel ixi(0)) processors. The version of Roo we used is based on OTTER 2.2xat (June 1992).

\section{Settings and Set of Support}

Within each set, all of the OTTER jobs used the same settings. However, the setting - for thr" basic set were substantially different from those for the equality set. The Roo jobs used settings slightly different from the OTTER jobs, and (for small technical reasons) the Roo settiugs for the basic set varied slightly, depending on whether equality is present.

For the basic set, the initial set of support consisted of the positive input clauses, excrept $(x=x)$. For the equality set, the initial set of support depended on whether the throrem has an obvious special hypothesis - if so, then the set of support was the special hypothesis and the denial of the conclusion; if not, the set of support consisted of all input clauses.

The rules for the equality set state that an ordering on the symbols may be inclucled with the input clauses. The ordering is used to orient equality literals.

\subsection{Settings for the Basic Set}

\begin{tabular}{lll} 
OTTER: basic set & Roo: basic with equality & Roo: basic without equality \\
\hline set(hyper_res) & set(index_for_back_demod) & \\
set(back_demod) & set(hyper_res) & set(hyper_res) \\
set(dynamic_demod_all) & set(back_demod) & \\
assign(pick_given_ratio,5) & assign(pick_given_ratio,5) & assign(pick_given_ratio,5) \\
clear(print_kept) & clear(print_kept) & clear(print_kept) \\
issign(max_mem,20000) & assign(max_mem,32000) & assign(max_mem,32000) \\
set(control_mernory) & set(control_mernory) & set(control_memory) \\
\hline
\end{tabular}

\subsection{Settings for the Equality Set}

\begin{tabular}{ll} 
()TTER: equality set & Roo: equality set \\
\hline set(knuth_bendix) & set(knuth_bendix) \\
set(index_for_back_demod) & set(index_for_back_demod) \\
sft(process_input) & set(process_input) \\
assign(max_mern,16000) & assign(max_mern,32000) \\
s.t(control_menory) & set(control_mernory) \\
st-1(lex_rpo) & set(lex_rpo) \\
cl.ar(print_kept) & clear(print_kept) \\
cliar(print_new_demod) & clear(print_new_demod) \\
cl.ar(print_bark_dernod) & clear(print_back_demod) \\
\hline
\end{tabular}

\subsection{Description of the Settings}

set(hyper_res). Thit option activates the inference rule hyperresolution.

set(back_demod). When new equalities are deduced, this option callses them to be nerel as rewrite rules. 
Table 1: Results for Basic Theorems

\begin{tabular}{|c|c|c|c|}
\hline & OTTER & Roo-8 & RoO- 12 \\
\hline \multicolumn{4}{|c|}{ Theorem 1: $x^{2}=\epsilon$ Group } \\
\hline proof time & 0.20 & 0.32 & 0.32 \\
\hline generated & 222 & 2300 & 1.867 \\
\hline kept & 13 & 30 & 40 \\
\hline memory $(\mathrm{K})$ & 31 & 728 & 564 \\
\hline \multicolumn{4}{|c|}{ Theorem 2: Commutator } \\
\hline proof time & 35.60 & 26.89 & 25.97 \\
\hline generated & 20575 & 88838 & 131429 \\
\hline kept & 4505 & 3684 & 1697 \\
\hline memory $(K)$ & 1564 & 12515 & 12670 \\
\hline \multicolumn{4}{|c|}{ Theorem $3: x^{2}=x$ Ring } \\
\hline proof time & 145.41 & 35.57 & 38.18 \\
\hline generated & 56025 & 134744 & 221890 \\
\hline kept & 13990 & 4316 & 2736 \\
\hline memory $(\mathrm{K})$ & 4342 & 14333 & $187: 39$ \\
\hline \multicolumn{4}{|c|}{ Theorem 4: XGK } \\
\hline proof time & 407.50 & 159.87 & 55.37 \\
\hline generated & 177109 & 663722 & 263233 \\
\hline kept & 15320 & 16519 & 9466 \\
\hline memory $(\mathrm{K})$ & 8047 & 19539 & 22189 \\
\hline \multicolumn{4}{|c|}{ Theorem 5: Imp-4 (CD-67) } \\
\hline proof time & 7711.98 & 1051.55 & 909.95 \\
\hline generated & 8341570 & 7171447 & $8182: 376$ \\
\hline kept & 17862 & 14855 & 17666 \\
\hline memory $(\mathrm{K})$ & 10729 & 13983 & 15098 \\
\hline \multicolumn{4}{|c|}{ Theorem 6: MV-1 (CD-57) } \\
\hline proof time & 17.68 & 4.37 & 14.71 \\
\hline generated & 16687 & 24159 & 114051 \\
\hline kept & 4837 & 1024 & 2000 \\
\hline memory $(\mathrm{K})$ & 2171 & 6479 & 12161 \\
\hline \multicolumn{4}{|c|}{ Theorem 7: MV-2 (CD-60) } \\
\hline proof time & 2184.96 & 427.89 & 152.53 \\
\hline generated & 3214280 & 4311090 & 1997084 \\
\hline kept & 16250 & 12374 & 10750 \\
\hline memory $(\mathrm{K})$ & 7216 & 13664 & 13755 \\
\hline
\end{tabular}


Table 2: Results for Equality Problems OtTer Roo-8 Roo- 12

\begin{tabular}{|c|c|c|c|}
\hline \multicolumn{4}{|c|}{ Theorem EQ-1: Commutator } \\
\hline proof time & 1.49 & 0.76 & 0.86 \\
\hline tod & 542 & 1727 & 2144 \\
\hline $\mathrm{t}$ & 114 & & 89 \\
\hline emory $(K)$ & 255 & 1208 & 1460 \\
\hline \multicolumn{4}{|c|}{ Theorem EQ-2: Robbins, $c+c=c$} \\
\hline proof time & 98.19 & 18.63 & 13.43 \\
\hline ated & 50001 & 560 & 59151 \\
\hline & 4548 & & 1235 \\
\hline ory $(K)$ & 5652 & 12676 & 13342 \\
\hline \multicolumn{4}{|c|}{ Theorem EQ-3: TBA } \\
\hline time & 16.78 & 4.10 & 3.16 \\
\hline ated & 3945 & 9307 & 11170 \\
\hline $\mathrm{k}$ & 1030 & 620 & 378 \\
\hline emory $(K)$ & 1564 & 4880 & 5043 \\
\hline \multicolumn{4}{|c|}{ Theorem EQ-4: Group single axiom } \\
\hline roof time & 44.12 & 10.56 & 9.25 \\
\hline ated & 3417 & 11778 & 16118 \\
\hline & 2507 & & 863 \\
\hline nory $(K)$ & 4470 & 13889 & 17110 \\
\hline \multicolumn{4}{|c|}{ Theorem EQ-5: Wajsberg algebra } \\
\hline f time & 2248.86 & 425.99 & 491.67 \\
\hline generated & 1012625 & 971543 & 1437272 \\
\hline & 5897 & 4374 & 4022 \\
\hline meinc & 6801 & 13376 & 14525 \\
\hline
\end{tabular}


set(dynamic_demod_all). This option has OTTER use all new orintable equalities as rewrite rules.

set(index_for_back_demod). This option causes indexing to be used when searching for terms to which a new rewrite rule can be applied. Roo requires this "option" whenever back demodulation is enabled. OTTER frequently benefits from this option.

assign(pick_given_ratio,5). By default OTTER chooses each new given clause based on its symbol count. Hence, a heavy clause that is needed for the proof cannot be used until all lighter clauses have been used. Recently we have found it useful to mix this strategy with a breadth-first strategy by choosing some percentage of the given clauses according to the order in which they are generated rather thar by weight. This setting chooses every sixth given clause in order of generation, and the rest by symbol count.

clear(print_kept). clear(print_new_demod).clear(print_back_demod). These options suppress output, saving file space and a little time.

$\operatorname{assign}\left(\max \_m e m, 20000\right)$. This setting restricts memory usage to 20 megabytes. Its real use is in conjunction with the next parameter.

set(control_memory). This setting has a relatively complex affect. Every ten given clauses, memory usage is analyzed. If more than a third of max mem has been used, then the max_weight parameter is automatically set to a value calculated such that only the lightest $5 \%$ of the clauses in the current set of support have lower weight. No clauses are cleleted, but from this point on, new clauses heavier than this weight are discarded. Using this parameter has the effect of allowing the system to choose a value for max_weight and adjust it during the run.

set(knuth_bendix). This option causes OTTER and Roo to automatically set a collection of options that approximate a Knuth-Bendix completion procedure. Under this option, the theorem prover orders equalities, paramodulates from left sides into left sides, and back demodulates.

set(process_input). This option causes all input clauses to be processed (subsumption, deriodulation, equality ordering, back demodulation) as if they were generated clauses.

lex(list of symbols). This command specifies an ordering on constant, function, and predicate symbols, with smallest first. For the experiments described in this paper, the ordering is used to attempt to orient equalities.

set(lex.rpo). This options specifies the lexicographic recursive path ordering for comparing terms when attempting to orient equalities.

Irpo_Ir_status(list of symbols). This command specifies that function symbols are to be compared left-to-right when applying the lexicographic recursive path ordering.

\section{Failures on Equality Theorems 6-10}

Theorem EQ-6. The fragnent $\{B, W, M\}$ of combinatory logic contains fixed point combinators. O'TTER found a prof, but the setting were different from those used in theorems EQ-1 through EQ-;. The important difference is that the initial sol, of 
support consists of the denial only (so that all generated clauses are negative), and paramodulation is allowed into both arguments of equality literals. The following input file causes OTTER to find a proof of EQ-6 in about 27 seconds.

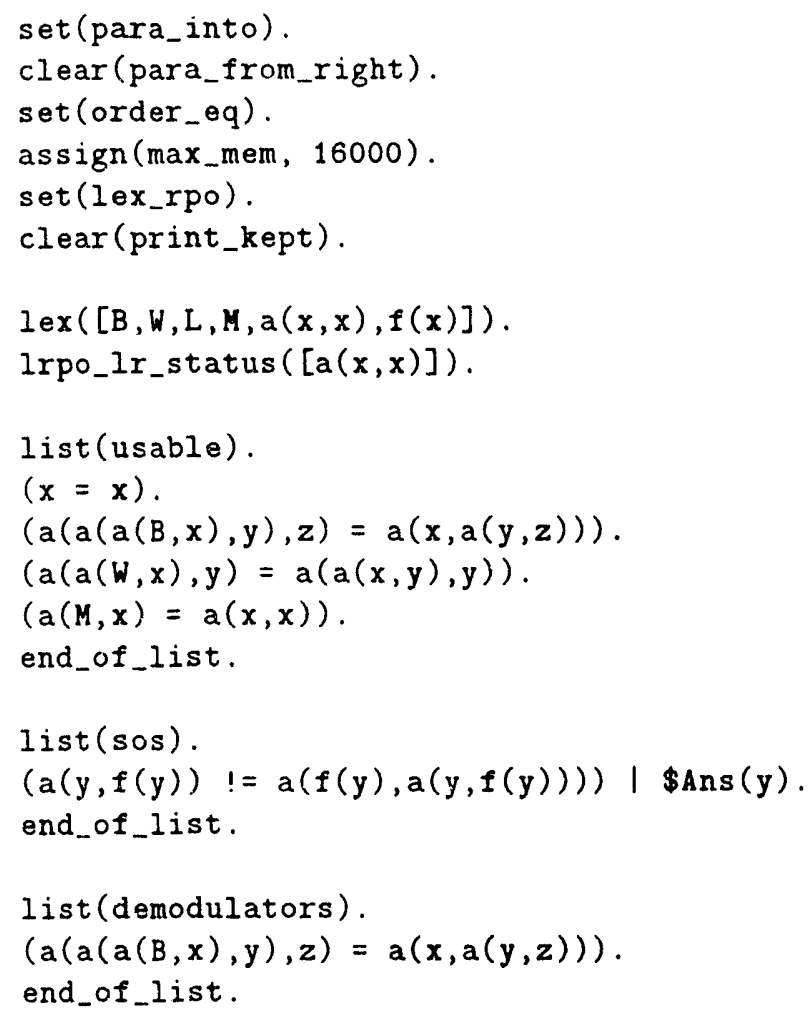

Theorem EQ-7. Rings in which $x^{3}=x$ are commutative. As far as we know, OTTFik has never found a proof of this theorem, except with highly specialized settings and weight templates. We suspect that with associative-commutative unification, OTTER would be able to prove it.

Theorem EQ-8. The fragment $\{B, W\}$ of combinatory logic contains fixed point combinators. This theorem is much more difficult than EQ-6, and the strategy above that works for EQ- 6 fails for EQ-8. The kernel method [7], which was developed for this type of problem, finds a proof of EQ-8 within a few seconds.

Theorems EQ-9 and EQ-10. On Moufang identities in nonassociative rings (EQ-9), and on right alternative nonassociative rings (EQ-10). The complicated definitions in these theorems cause terms in the conclusion to be greatly expanded. OTTER cannot cope with the complrx conclusions, because it likes to focus on simple terms. As with (EQ7), we believe that associative-commutative unification would be helpful for these theorems.

\section{Summary of ()TTER Outputs for the Basic Set}

\subsection{Theorem 1: $l^{2}=e$ Groups are Commutative (P-form)}


The job began on altair.mcs.anl.gov, Wed Jun 3 13:15:19 1992

The command was "otter22".

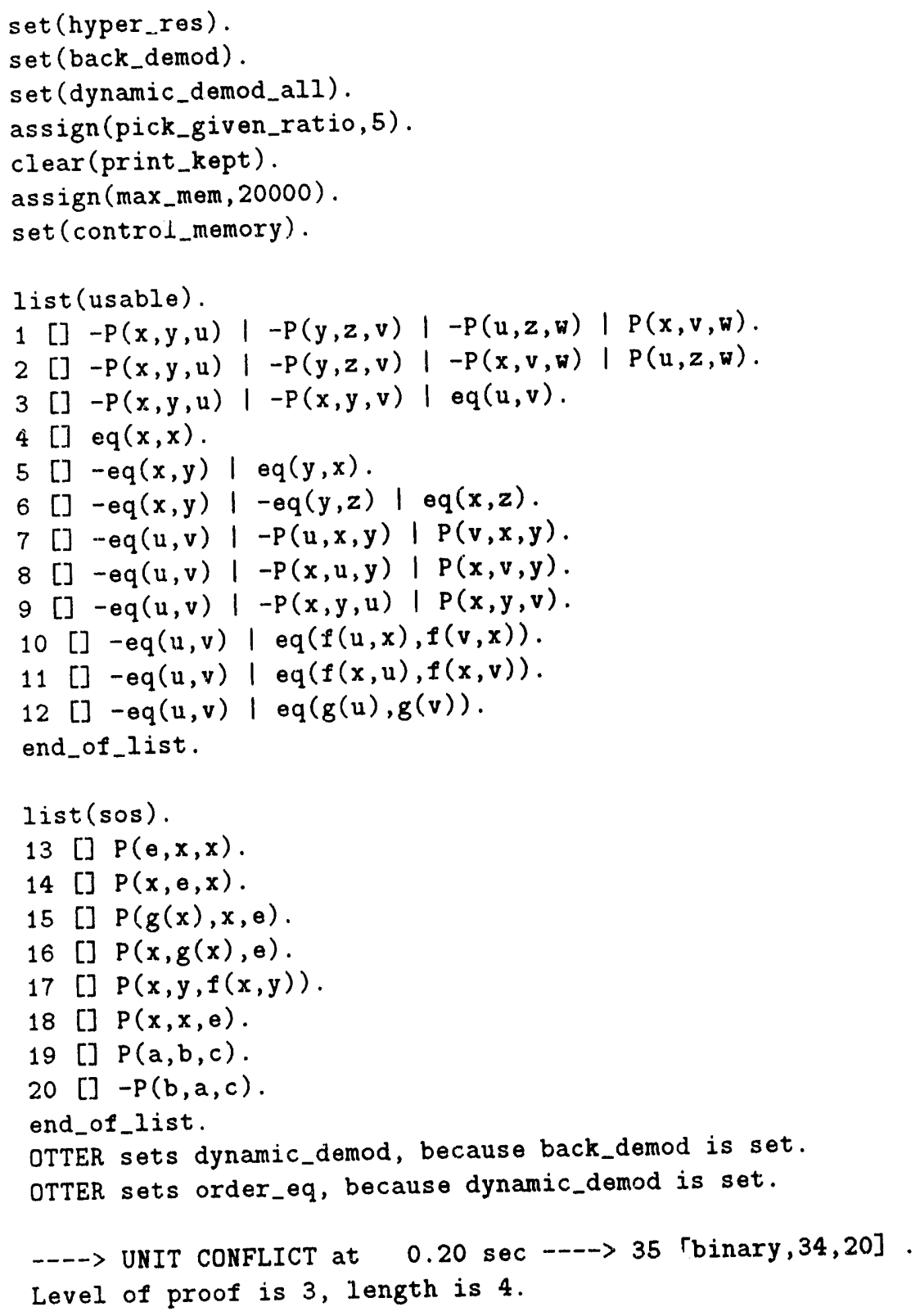

1[]$-P(x, y, u)|-P(y, z, v)|-P(u, z, w) \mid P(x, v, w)$.

2[]$-P(x, y, u)|-P(y, z, v)|-P(x, v, w) \mid P(u, z, w)$.

13[]$P(e, x, x)$.

14[]$P(x, e, x)$.

18 [] $P(x, x, e)$.

19 [] $P(a, b, c)$.

20[]$-P(b, a, c)$.

21 [hyper, 19,2,18,14] $P(c, b, a)$.

22 [hyper, 19, 1,18,13] $P(a, c, b)$.

23 [hyper, $21,1,18,13] P(c, a, b)$. 
34 [hyper, 23, 2, 22,19] $P(b, a, c)$.

35 [binary, 34, 20].

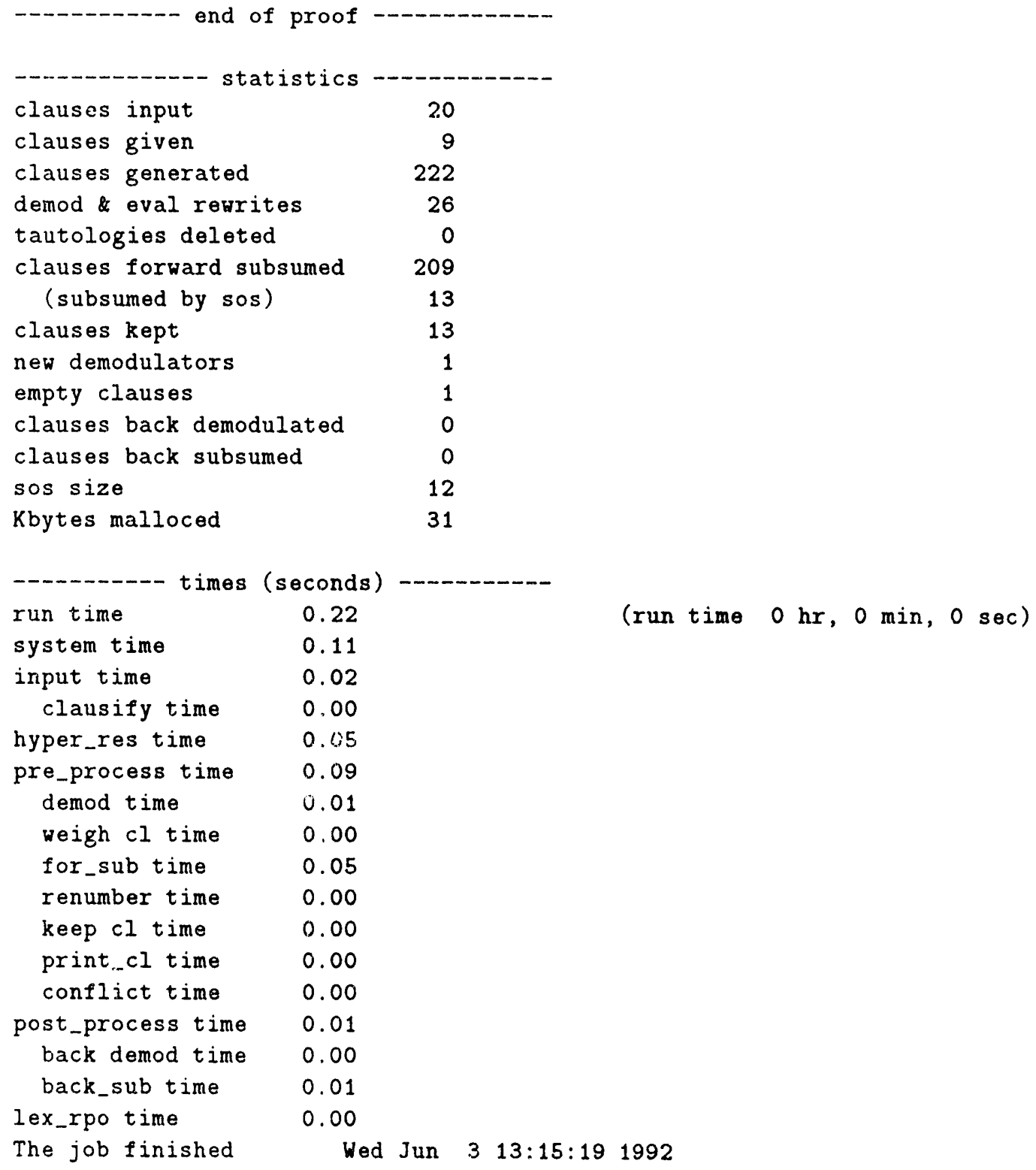

\subsection{Theorem 2: The Commutator Theorem (P-form)}

-.-- OTTER 2.2, July 1991 -..-

The job began on altair.mcs.anl.gov, Wed Jun 3 13:13:02 1992

The command was "otter 22 ".

set (hyper_res).

$s \in t$ (back_demod).

set (dynamic_demod_all).

assign(pick_given_ratio, 5).

clear(print_kept).

assign (max_mem, 20000). 


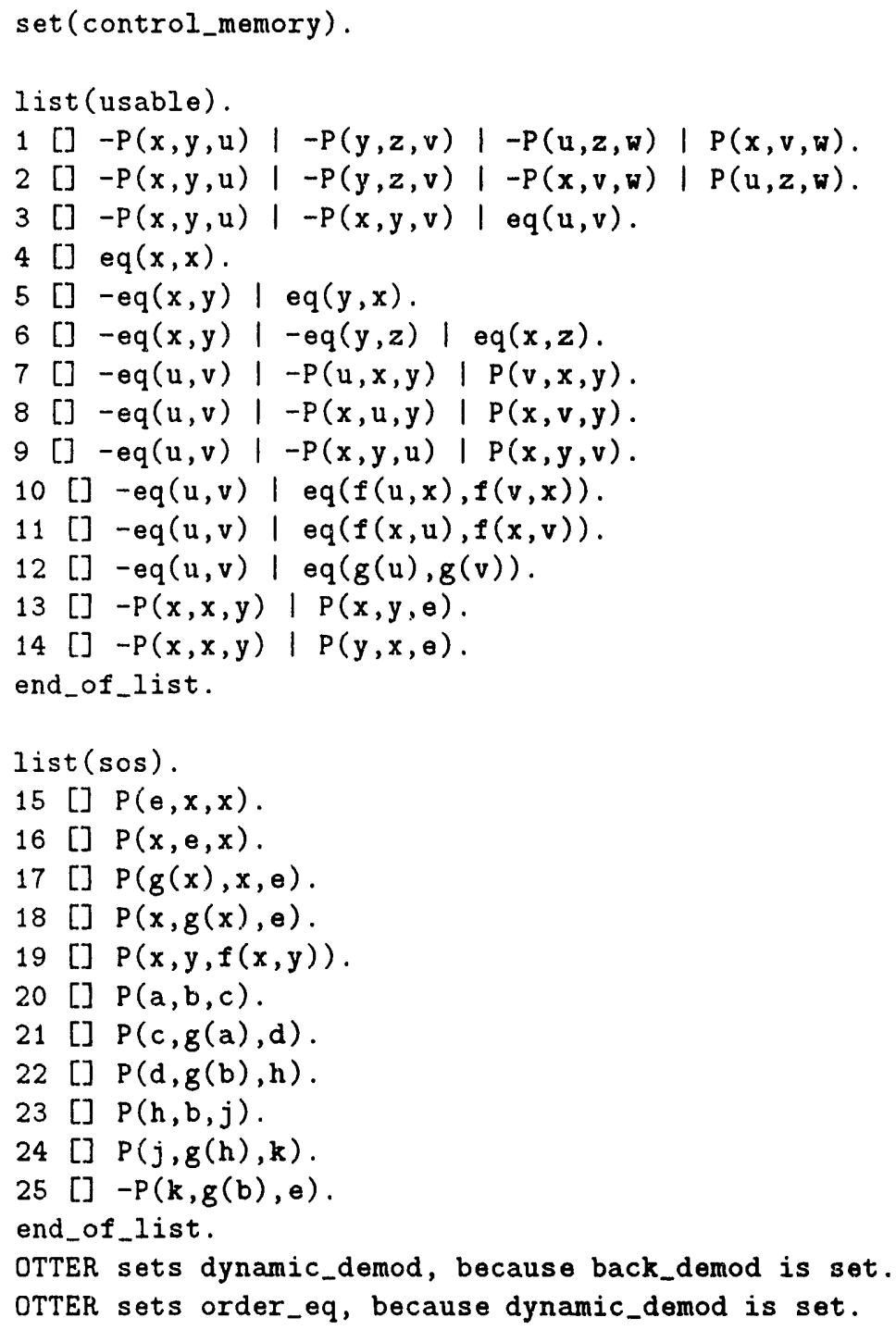

1[]$-P(x, y, u)|-P(y, z, v)|-P(u, z, w) \mid P(x, v, w)$.

2[]$-P(x, y, u)|-P(y, z, v)|-P(x, v, w) \mid P(u, z, w)$.

3[]$-P(x, y, u)|-P(x, y, v)|$ eq $(u, v)$.

9[]$-e q(u, v)|-P(x, y, u)| P(x, y, v)$.

13[]$-P(x, x, y) \mid P(x, y, e)$.

14[]$-P(x, x, y) \mid P(y, x, e)$.

15[]$P(e, x, x)$.

16[]$P(x, \theta, x)$.

17[]$P(g(x), x, e)$.

18 [] $P(x, g(x), e)$.

19 [] $P(x, y, f(x, y))$.

20 [] $P(a, b, c)$.

21 [] $P(c, g(a), d)$.

22 [] $P(d, g(b), h)$. 


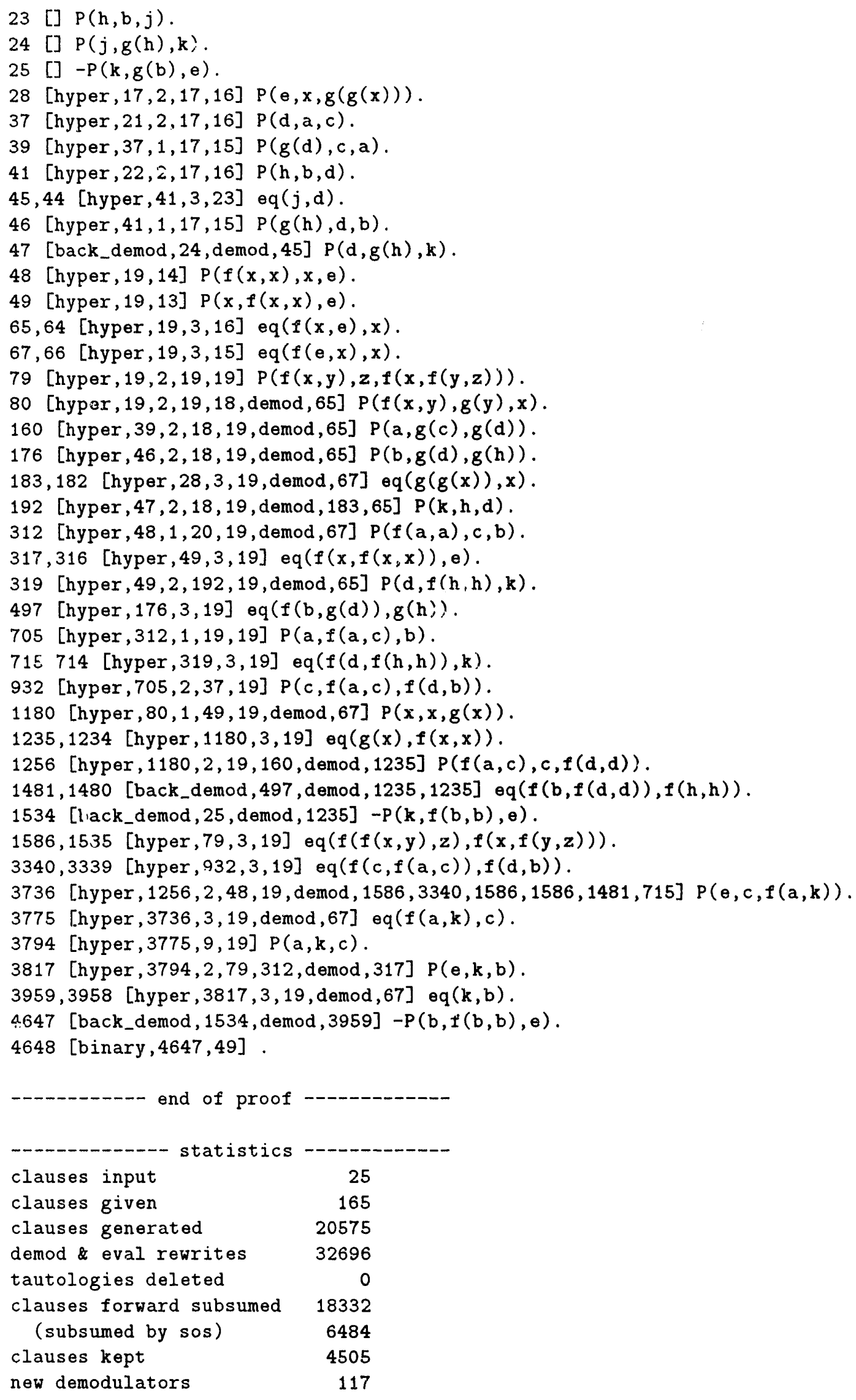




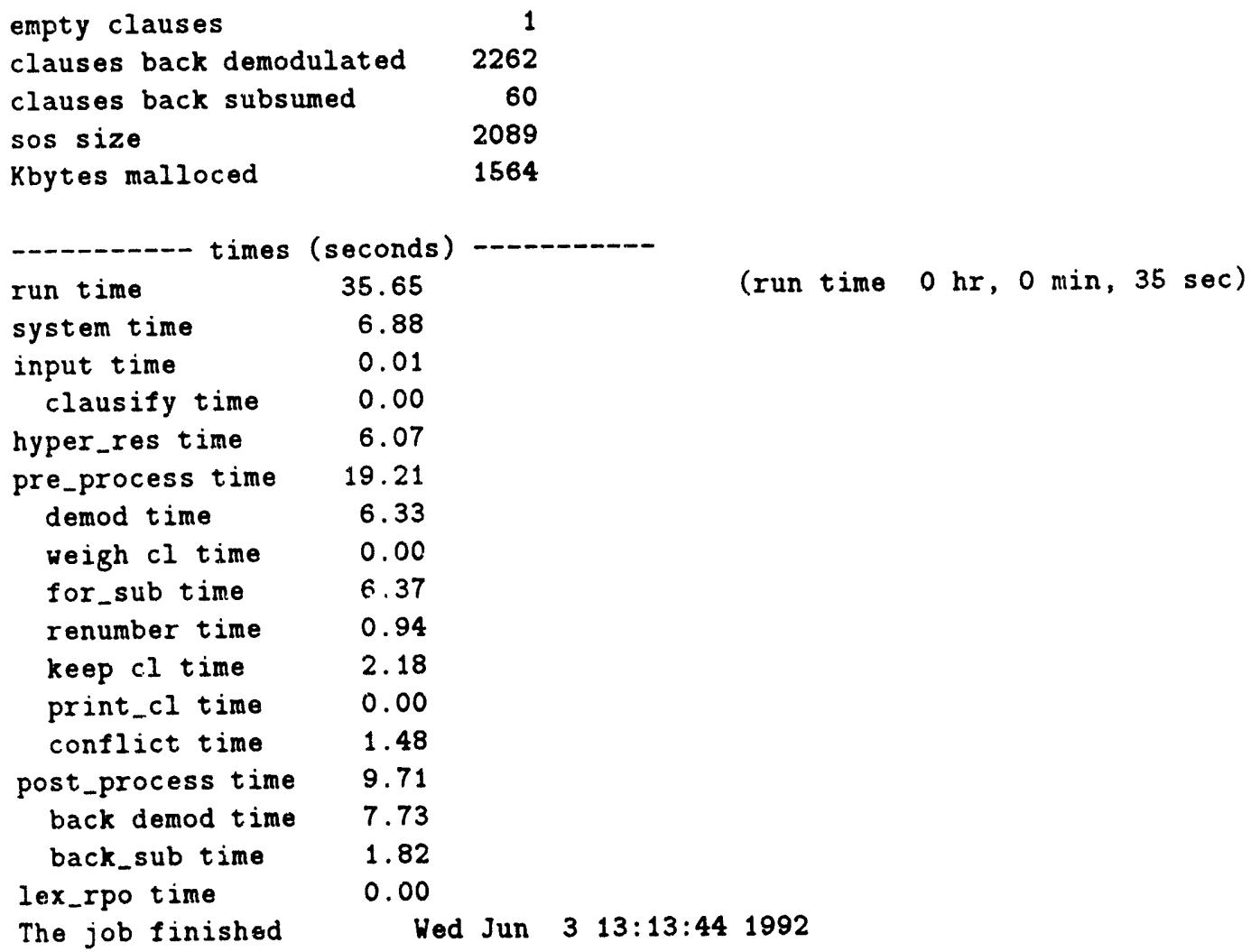




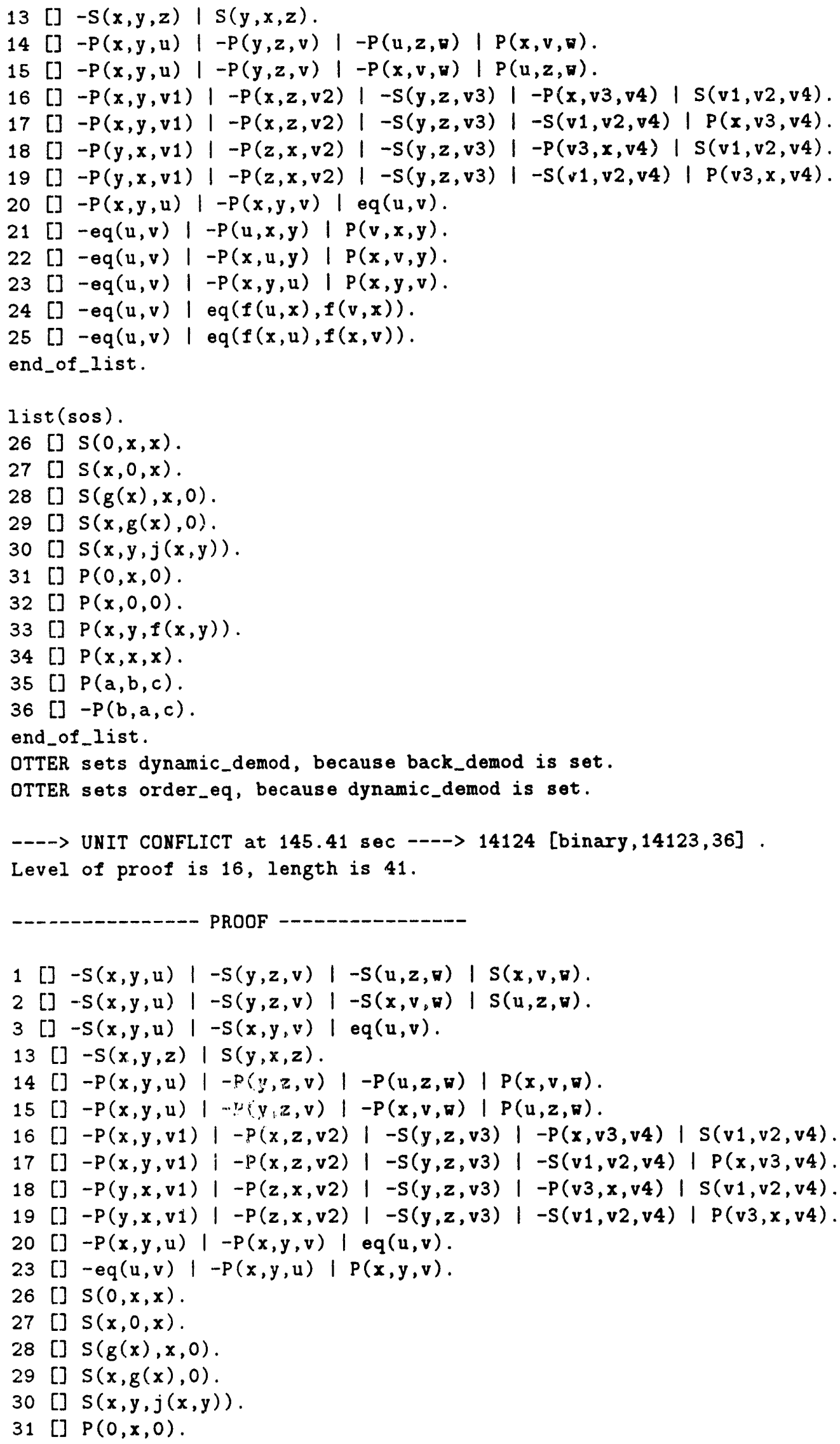

1[]$-S(x, y, u)|-S(y, z, v)|-S(u, z, \nabla) \mid S(x, v, w)$.

2[]$-S(x, y, u)|-S(y, z, v)|-S(x, v, w) \mid S(u, z, w)$.

3[]$-S(x, y, u)|-S(x, y, v)|$ eq $(u, v)$.

13[]$-S(x, y, z) \mid S(y, x, z)$.

14[]$-P(x, y, u)|-P(y, z, v)|-P(u, z, w) \mid P(x, v, w)$.

15[]$-P(x, y, u)|\cdots(y, z, v)|-P(x, v, w) \mid P(u, z, \nabla)$.

16[]$-P(x, y, v 1)|-P(x, z, v 2)|-S(y, z, v 3)|-P(x, v 3, v 4)| S(v 1, v 2, v 4)$.

17[]$-P(x, y, v 1)|-P(x, z, v 2)|-S(y, z, v 3)|-S(v 1, v 2, v 4)| P(x, v 3, v 4)$.

18[]$-P(y, x, v 1)|-P(z, x, v 2)|-S(y, z, v 3)|-P(v 3, x, v 4)| S(v 1, v 2, v 4)$.

19[]$-P(y, x, v 1)|-P(z, x, v 2)|-S(y, z, v 3)|-S(v 1, v 2, v 4)| P(v 3, x, v 4)$.

20[]$-P(x, y, u)|-P(x, y, v)|$ eq $(u, v)$.

23[]$-e q(u, v)|-P(x, y, u)| P(x, y, v)$.

26 [] $s(0, x, x)$.

27 [] $S(x, 0, x)$.

28 [] $S(g(x), x, 0)$.

29 [] $S(x, g(x), 0)$.

30 [] $S(x, y, j(x, y))$.

31 [] $P(0, x, 0)$. 


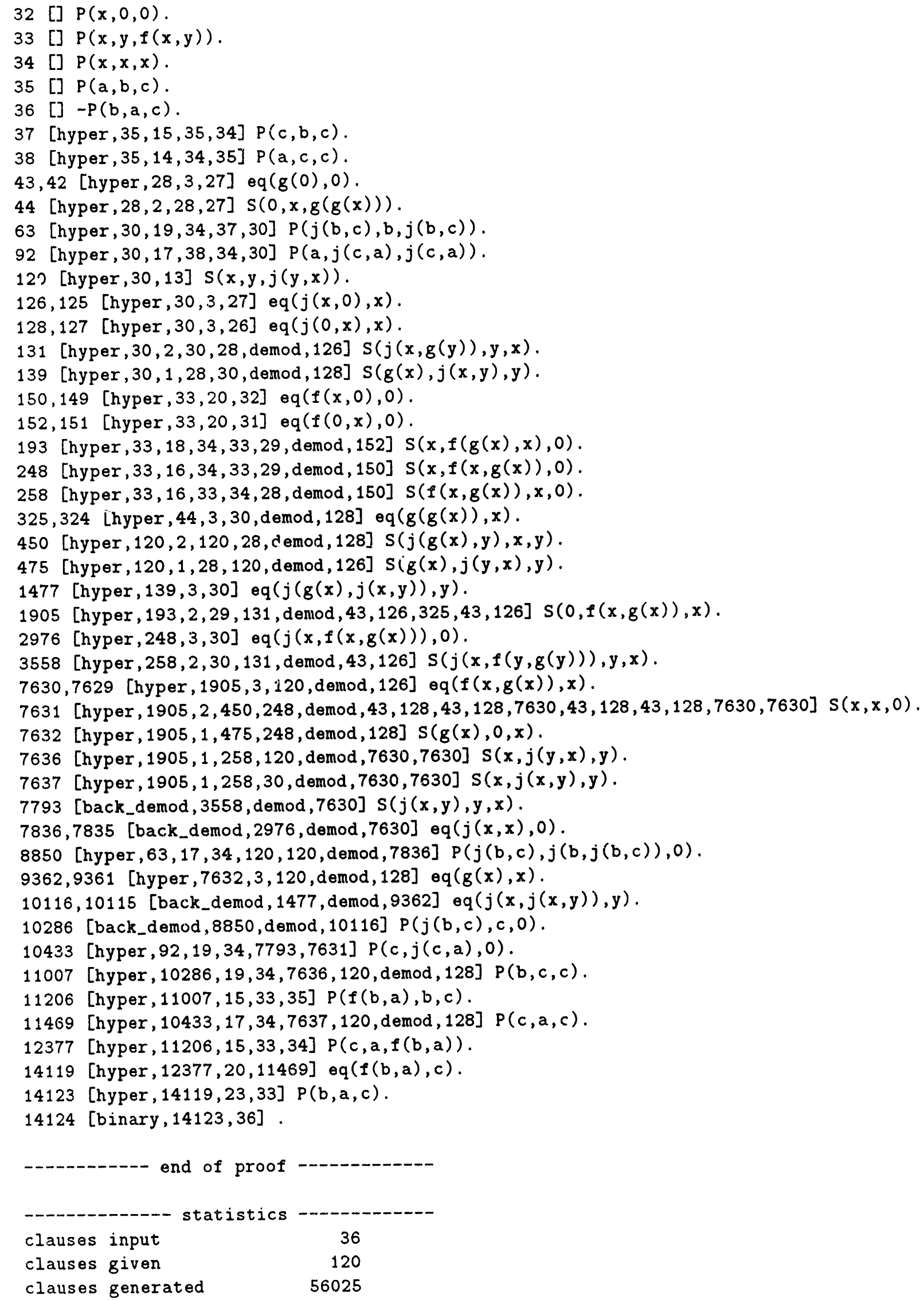




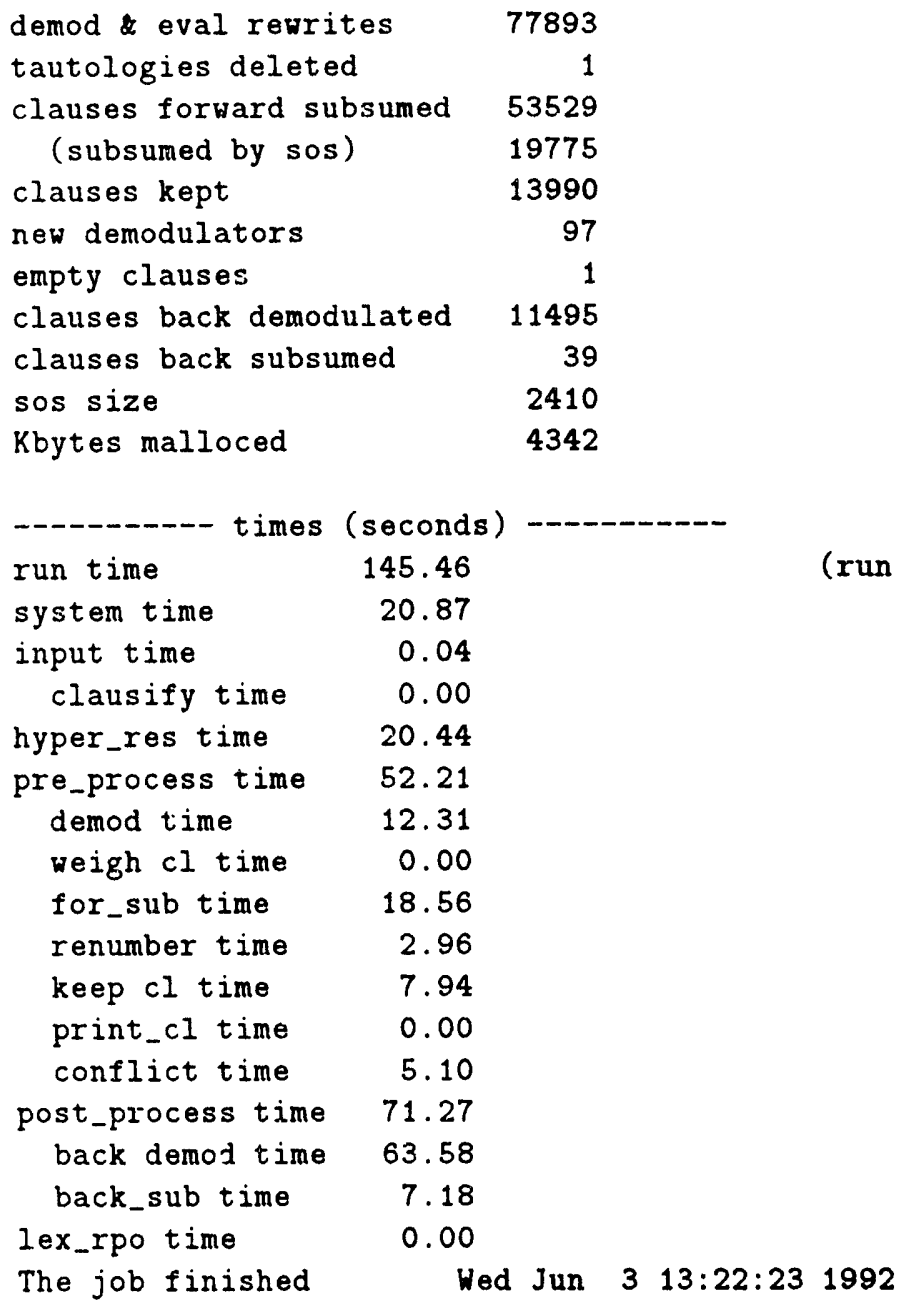

\subsection{Theorem 4: Equivalential Calculus, XGK $\rightarrow$ F'YO}

OTTER 2.2, July 1991

The job began on altair.mcs.anl.gov, Hed Jun 3 13:22:37 1992

The command was "otter 22 ".

set (hyper_res).

set (back_demod).

set (dynamic_demod_all).

assign(pick_given_ratio, 5 ).

clear (print_kept).

assign (max_mem, 20000).

set (control_memory).

list (usable).

1 [] $-P(x)|-P(e(x, y))| P(y)$.

end_of_list.

list (sos).

2 [] $P(e(x, e(e(y, e(z, x)), e(z, y))))$.

3 [] $-P(e(e(e(a, e(b, c)), c), e(b, a)))$. 
end_of_Iist.

OTTER sets dynamic_demod, because back_demod is set.

OTTER sets order_eq, because dynamic_demod is set.

Resetting weight limit to 20 .

-- > UNIT CONFLICT at $407.50 \mathrm{sec}--->15324$ [binary, 15323,3].

Level of proof is 13 , length is 19 .

1[]$-P(x)|-P(e(x, y))| P(y)$.

2[]$P(e(x, e(e(y, e(z, x)), e(z, y))))$.

3[]$-P(e(e(e(a, e(b, c)), c), e(b, a)))$.

4 [hyper, 2,1,2] $P(e(e(x, e(y, e(z, e(e(u, e(v, z)), e(v, u))))), e(y, x)))$.

6 [hyper, 4,1,2] $P(e(e(e(e(x, e(y, z)), e(y, x)), \theta(z, u)), u))$.

8 [hyper, $6,1,6] \mathrm{P}(\mathrm{e}(\mathrm{x}, \mathrm{x}))$.

9 [hyper, $6,1,4] P(e(e(x, e(e(y, e(z, x)), e(z, y))), e(u, u)))$.

13 [hyper $, 8,1,2] P(e(e(x, e(y, e(z, z))), e(y, x)))$.

18 [hyper $13,1,2] P(e(e(x, e(x, y)), y))$.

21 [hyper $, 13,1,2] P(e(e(x, e(y, e(e(z, e(u, e(v, v))), e(u, z)))), e(y, x)))$.

39 [hyper, 18,1,13] $P(e(x, e(y, e(y, e(x, e(z, z))))))$.

42 [hyper, 18,1,2] $P(e(e(x, e(y, e(e(z, e(z, u)), u))), e(y, x)))$.

108 [hyper, 39,1,4] $P(e(x, \theta(y, e(y, x))))$.

133 [hyper, 108,1,2] $P(e(e(x, e(y, e(z, e(u, e(u, z))))), e(y, x)))$.

146 [hyper, $9,1,2] P(e(e(x, e(y, e(e(z, e(e(u, e(v, z)), e(v, u))), e(w, w)))), e(y, x)))$.

682 [hyper $, 42,1,18] P(e(x, e(y, e(y, e(x, e(e(z, e(z, u)), u))))))$.

2253 [hyper, 133,1,2] $P(e(e(e(x, e(x, y)), e(y, z)), z))$.

8738 [hyper, 682,1,4] $P(e(x, e(y, e(e(z, e(z, y)), x))))$.

8897 [hyper, 8738,1,2253] $P(e(e(x, e(x, y)), e(z, e(z, y))))$.

9048 [hyper, 8897,1,21] $P(e(e(x, e(y, e(z, z))), e(u, e(u, e(y, x)))))$.

13855 [hyper, 9048, 1,4] $P(e(x, e(e(y, z), e(e(z, e(y, x)), e(u, u)))))$.

15323 [hyper, 13855,1,146] $P(e(e(e(x, e(y, z)), z), e(y, x)))$.

15324 [binary, 15323, 3].

$\begin{array}{lr} & \\ \text { clauses input } & 3 \\ \text { clauses given } & 587 \\ \text { clauses generated } & 177109 \\ \text { demod \& eval rewrites } & 0 \\ \text { clauses wt, lit, sk delete } & 102987 \\ \text { tautologies deleted } & 0 \\ \text { clauses forward subsumed } & 58802 \\ \quad \text { (subsumed by sos) } & 12239 \\ \text { clauses kept } & 15320 \\ \text { new demodulators } & 0 \\ \text { empty clauses } & 1 \\ \text { clauses back demodulated } & 0 \\ \text { clauses back subsumed } & 0 \\ \text { sos size } & 14735 \\ \text { Kbytes malloced } & 8047\end{array}$

times (seconds) 


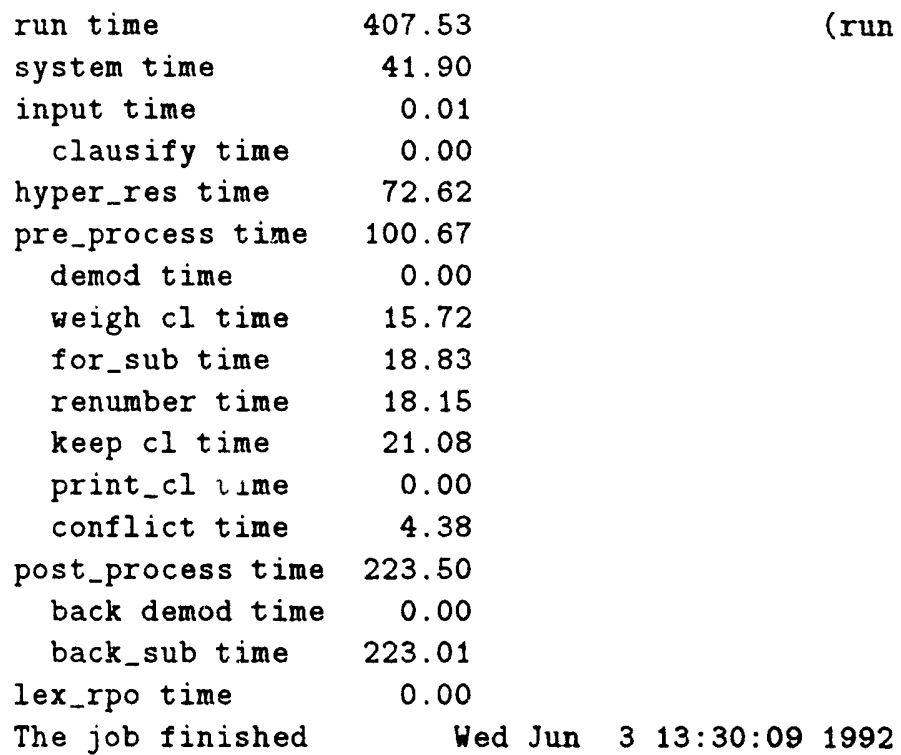

\subsection{Theorem 5: Implicational Calculus Single Axiom, CD-67 (Imp-4)}

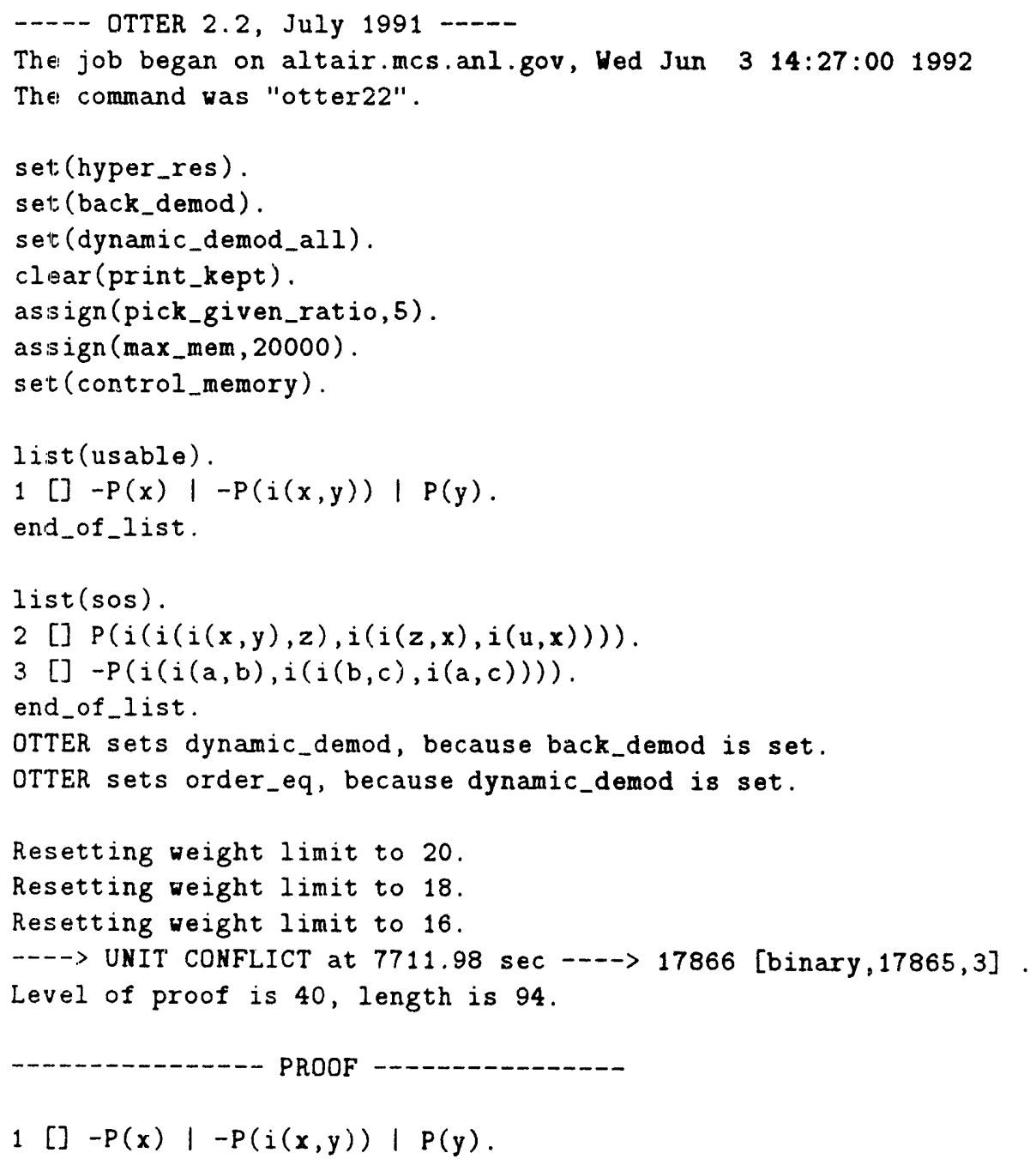




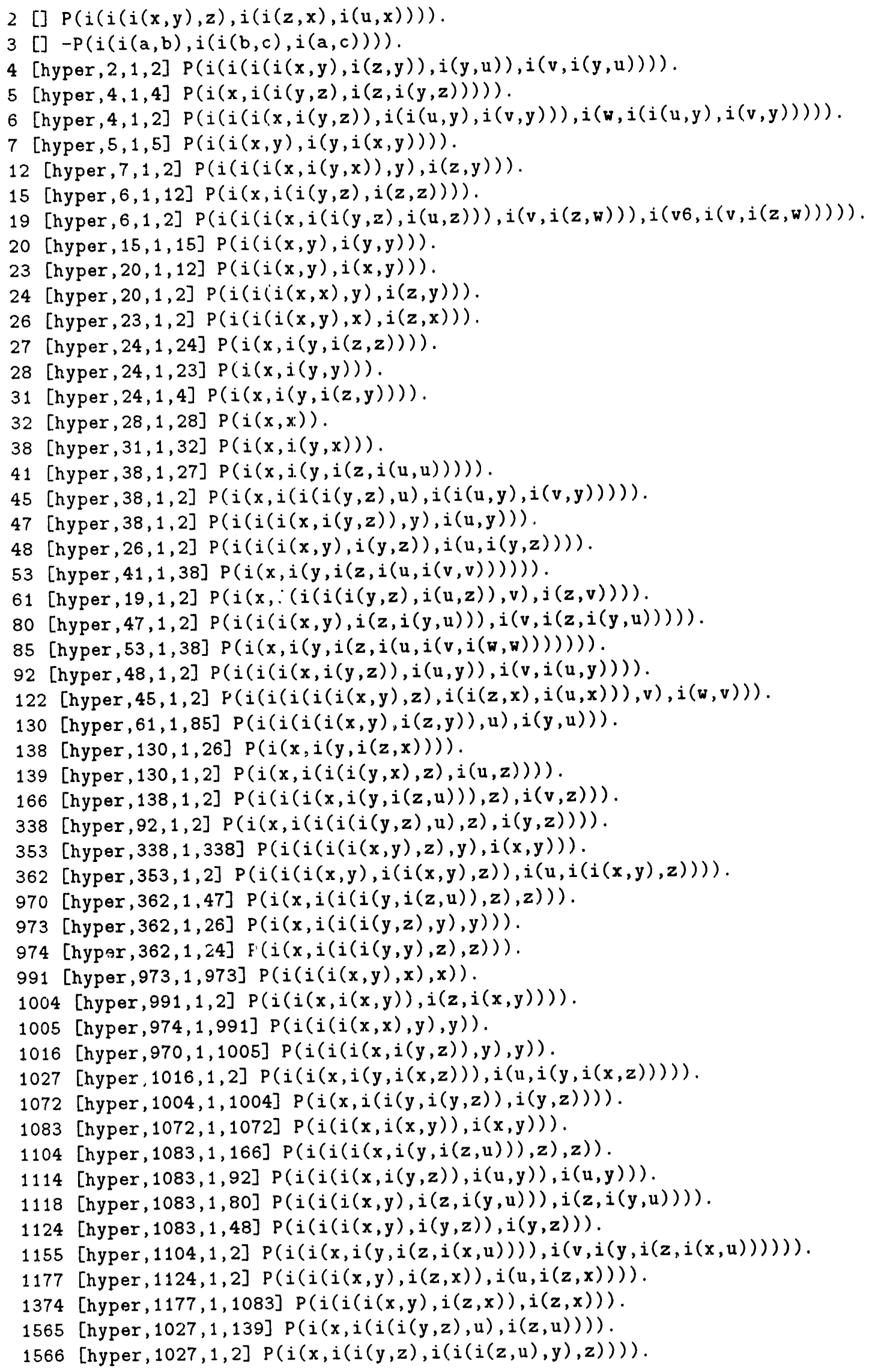


1567 [hyper, 1027, 1, 1083] $P(i(i(x, i(y, i(x, z))), i(y, i(x, z))))$.

1577 [hyper, $1565,1,1565] P(i(i(i(x, y), z), i(y, z)))$.

1588 [hyper, $1577,1,2] P(i(x, i(i(x, y), i(z, y))))$.

1592 [hyper, 1577, 1,122] $P(i(x, i(y, i(i(y, z), i(u, z)))))$.

1645 [hyper, $1588,1,2]$ P( $(i(i(i(i(i(x, y), z), i(u, z)), x), i(v, x)))$.

1661 [hyper, 1566, 1,1592] $P(i(i(x, y), i(i(i(y, z), x), y)))$.

1672 [hyper, 1661, 1,1588] $P(i(i(i(i(i(x, y), i(z, y)), u), x), i(i(x, y), i(z, y))))$.

1703 [hyper, 1661,1,1004] $P(i(i(i(i(x, i(y, z)), u), i(y, i(y, z))), i(x, i(y, z))))$.

1741 [hyper, 1661,1,138] $P(i(i(i(i(x, i(y, z)), u), z), i(x, i(y, z))))$.

1762 [hyper, 1661, 1,47] $P(i(i(i(i(x, y), z), i(i(u, i(y, v)), y)), i(x, y)))$.

1765 [hyper, 1661,1,26] $P(i(i(i(i(x, y), z), i(i(y, u), y)), i(x, y)))$.

2492 [hyper, 1645,1,1083] $P(i(i(i(i(i(x, y), z), i(u, z)), x), x))$.

4636 [hyper, 1762,1,2492] $P(i(i(i(i(i(x, i(i(y, z), u)), i(y, z)), v), z), i(y, z)))$.

7184 [hyper, 1155,1,1083] $P(i(i(x, i(y, i(z, i(x, u)))), i(y, i(z, i(x, u)))))$.

10842 [hyper, 4636,1,1765] $P(i(i(i(x, i(i(i(y, z), y), u)), i(i(y, z), y)), y))$.

10924 [hyper, 10842,1,1672] $P(i(i(x, y), i(i(i(x, z), x), y)))$.

10927 [hyper, 10924,1,1588] $P(i(i(i(x, y), x), i(i(x, z), i(u, z))))$.

10951 [hyper, 10927,1,1741] $P(i(x, i(y, i(i(x, z), i(u, z)))))$.

10953 [hyper, 10927, 1, 1703] $P(i(x, i(i(x, y), y)))$.

11237 [hyper, 10953,1,10924] $P(i(i(i(x, y), x), i(i(x, z), z)))$.

11252 [hyper, 10953,1,1661] $P(i(i(i(i(i(x, y), y), z), x), i(i(x, y), y)))$.

11310 [hyper, 10951,1,1577] $P(i(x, i(y, i(i(i(z, x), u), i(v, u)))))$.

11344 [hyper, $11237,1,1741] \quad P(i(x, i(y, i(i(x, z), z))))$.

11355 [hyper, $11237,1,2] P(i(i(i(i(x, y), y), i(x, z)), i(u, i(x, z))))$.

11414 [hyper, $11344,1,1577] P(i(x, i(y, i(i(i(z, x), u), u))))$.

12034 [hyper, $\left.11355,1,1^{\prime} 33\right] P(i(i(i(i(x, y), y), i(x, z)), i(x, z)))$.

12131 [hyper, 12034,1,11414] $P(i(x, i(i(i(y, i(i(x, z), z)), u), u)))$.

12134 [hyper, $12034,1,11310$ ] $P(i(x, i(i(i(y, i(i(x, z), z)), u), i(v, u))))$.

12136 [hyper, $12034,1,10951] P(i(x, i(i(i(i(x, y), y), z), i(u, z))))$.

12188 [hyper, 12131,1,1374] $P(i(i(i(x, i(i(i(y, z), u), u)), y), y))$.

12191 [hyper, 12131,1,1114] $P(i(i(i(x, i(i(i(y, i(z, u)), v), v)), z), z))$.

12238 [hyper, $12136,1,1567] P(i(i(i(i(x, y), y), z), i(x, z)))$.

12442 [hyper, 12188,1,11252] $P(i(i(x, i(i(i(x, y), z), z)), i(i(i(x, y), z), z)))$.

13088 [hyper, 12134,1,1118] $P(i(i(i(x, i(i(i(y, z), u), u)), v), i(z, v)))$.

13109 [typer, 12191,1,1672] $P(i(i(x, y), i(i(i(z, i(x, u)), y), y)))$.

13927 [hyper, 13088,1,1672] $P(i(i(i(x, y), z), i(i(i(u, x), z), z)))$.

14592 [hyper, 12442,1,13109] $P(i(i(i(i(x, y), i(x, z)), y), y))$.

14632 [hyper, 14592, 1,13927] $P(i(i(i(x, i(i(y, z), i(y, u))), z), z))$.

14829 [hyper, 14632, 1, 1672] $P(i(i(x, i(y, z)), i(i(y, x), i(y, z))))$.

15113 [hyper, $14829,1,10951] P(i(i(x, y), i(x, i(i(y, z), i(u, z)))))$.

16490 [hyper, $15113,1,12238$ ] $P(i(x, i(i(x, y), i(i(y, z), i(u, z)))))$.

17865 [hyper, 16490,1,7184] $P(i(i(x, y), i(i(y, z), i(x, z))))$.

17866 [binary, 17865, 3].

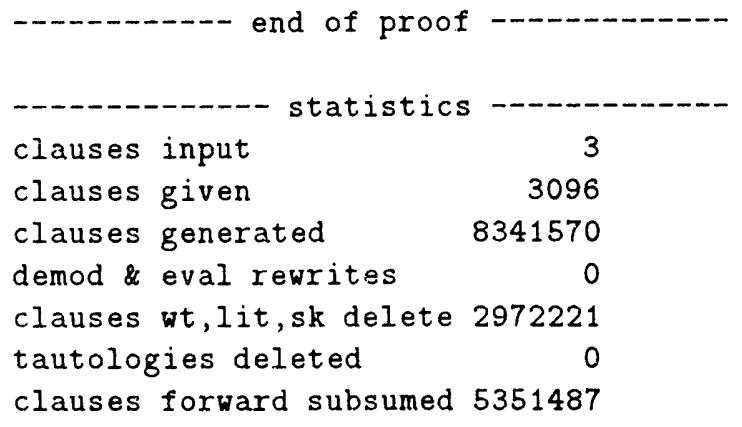




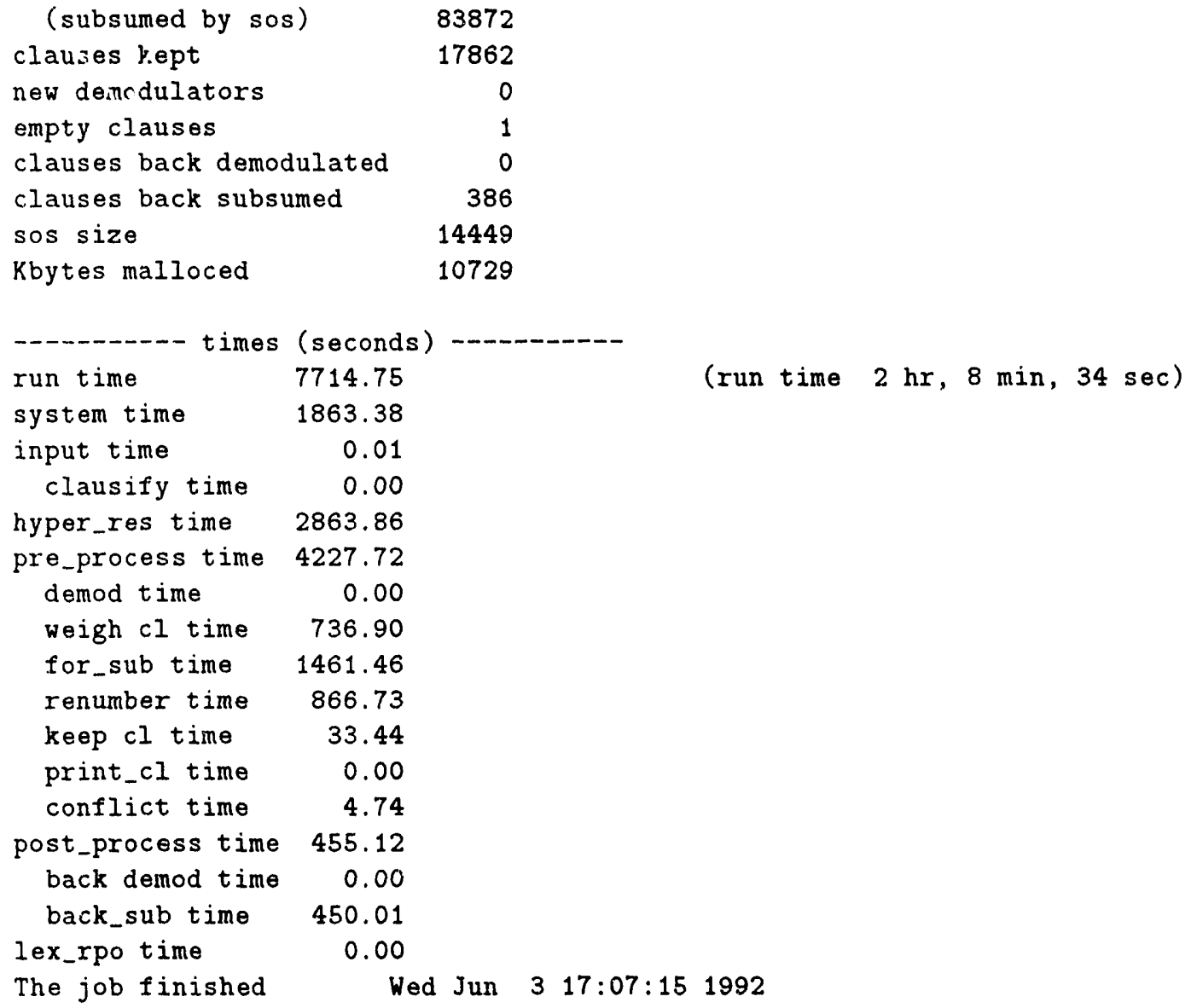

\subsection{Theorem 6: Many-valued Sentential Calculus, CD-57}

-.- OTTER 2.2, July 1991 -.--

The job began on altair.mcs.anl.gov, Wed Jun 3 13:16:52 1992

The command was "otter22".

set (hyper_res).

set (back_demod).

set (dynamic_demod_all).

clear(print_kept).

assign (pick_given_ratio, 5).

assign (max_mem, 20000).

set (control_memory).

list (usable).

1[]$-P(x)|-P(i(x, y))| P(y)$.

end_of_list.

list (sos).

2 [] $P(i(x, i(y, x)))$.

3 [] $P(i(i(x, y), i(i(y, z), i(x, z))))$.

4 [] $P(i(i(i(x, y), y), i(i(y, x), x)))$.

5 [] $P(i(i(n(x), n(y)), i(y, x)))$.

6[]$-P(i(i(a, b), i(i(c, a), i(c, b))))$. 
end_of_list.

OTTER sets dynamic_demod, because back_demod is set.

OTTER sets order_eq, because dynamic_demod is set.

$-\rightarrow->$ UNIT CONFLICT at $17.68 \mathrm{sec}-\rightarrow-\rightarrow 4844$ [binary, 4843,6].

Level of proof is 4 , length is 5 .

1[]$-P(x)|-P(i(x, y))| P(y)$.

2[]$P(i(x, i(y, x)))$.

3[]$P(i(i(x, y), i(i(y, z), i(x, z))))$.

4[]$P(i(i(i(x, y), y), i(i(y, x), x)))$.

6[]$-P(i(i(a, b), i(i(c, a), i(c, b))))$.

14 [hyper, $3,1,3] P(i(i(i(i(x, y), i(z, y)), u), i(i(z, x), u)))$.

15 [hyper, $3,1,2] P(i(i(i(x, y), z), i(y, z)))$.

24 [hyper $, 4,1,15] P(i(x, i(i(x, y), y)))$.

49 [hyper, 24,1,3] $P(i(i(i(i(x, y), y), z), i(x, z)))$.

4843 [hyper $49,1,14] P(i(i(x, y), i(i(z, x), i(z, y))))$.

4844 [binary, 4843,6 ] .

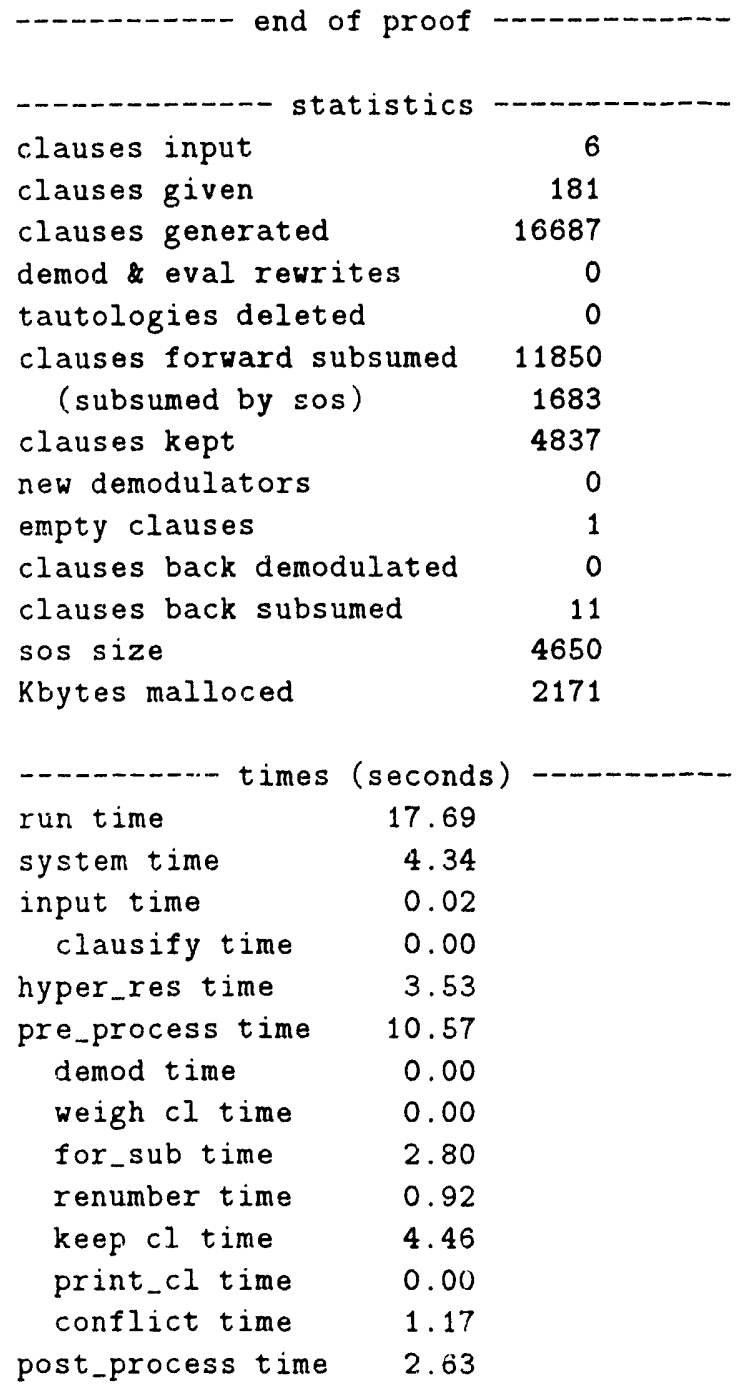




$\begin{array}{cl}\text { back demod time } & 0.00 \\ \text { back_sub time } & 2.57 \\ \text { lex_rpo time } & 0.00\end{array}$

The job finished
0.00

0.00

Wed Jun $3 \quad 13: 17: 141992$

\subsection{Theorem 7: Many-valued Sentential Calculus, CD-60}

OTTER 2.2, July 1991

The job began on altair.mcs.anl.gov, Wed Jun 3 13:31:24 1992

The command was "otter'22".
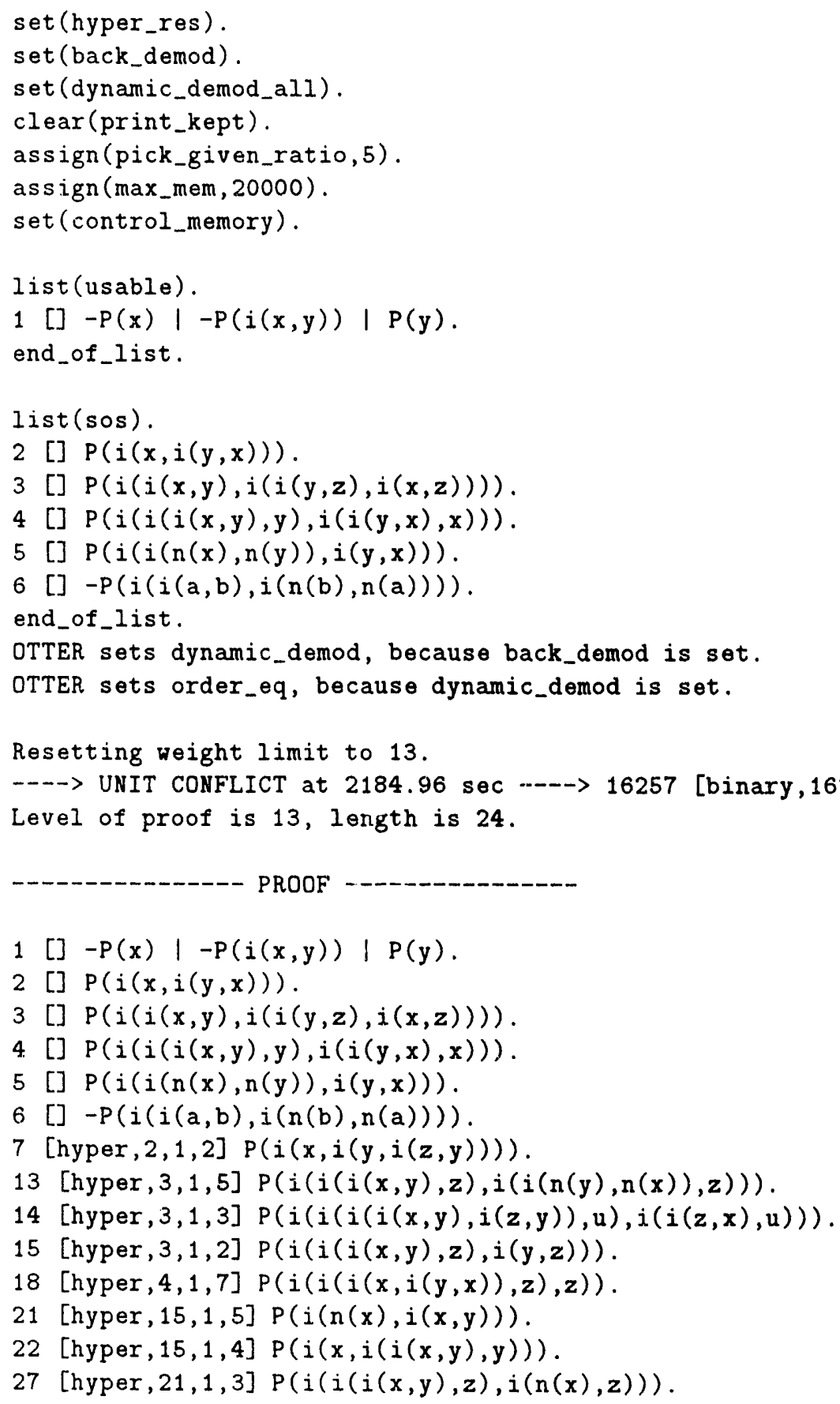


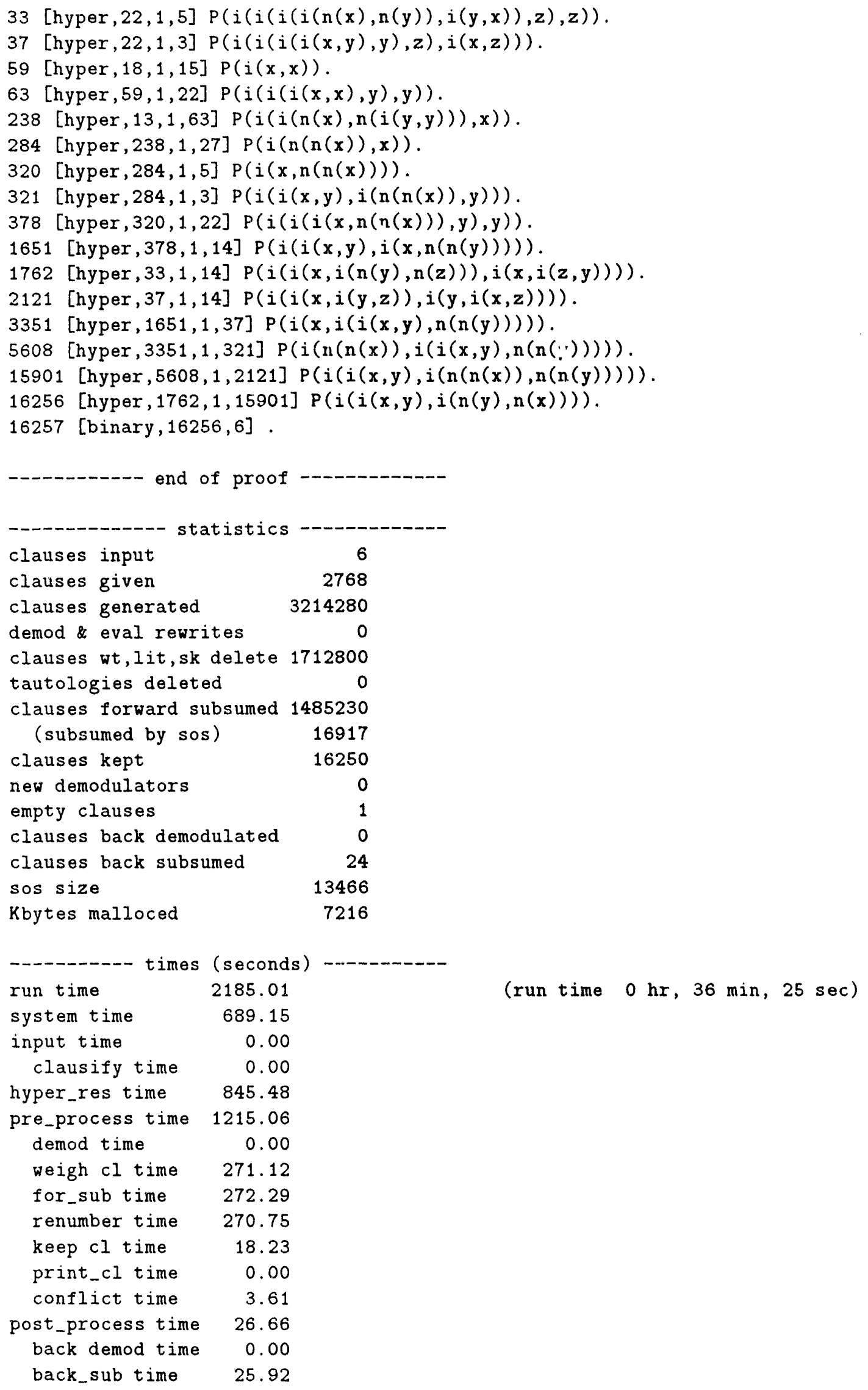


lex_rpo time $\quad 0.00$

The job finished Wed Jun 3 14:26:09 1992

\section{Summary of OTtER Outputs for the Equality Set}

\subsection{Theorem EQ-1: The Commutator Theorem}

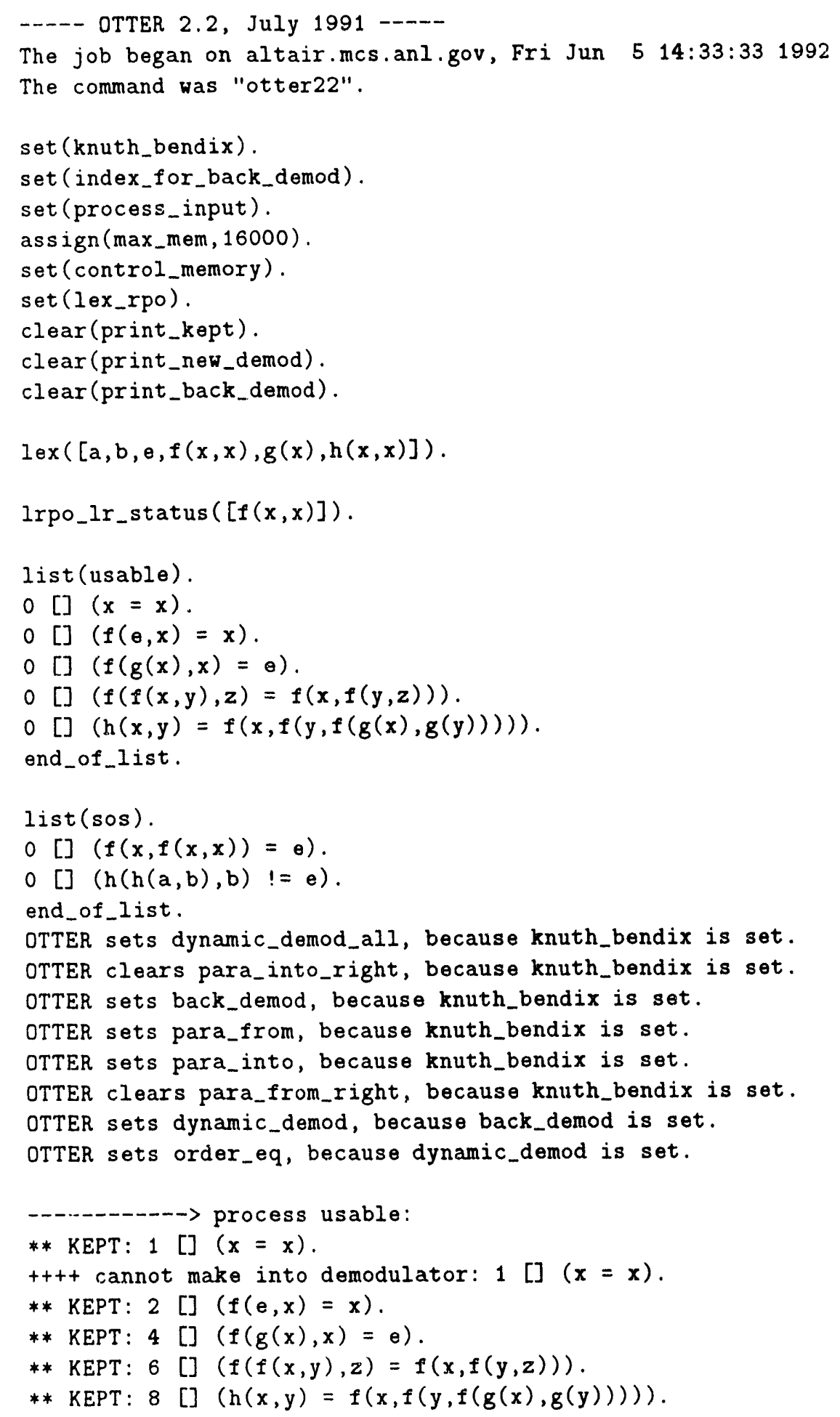




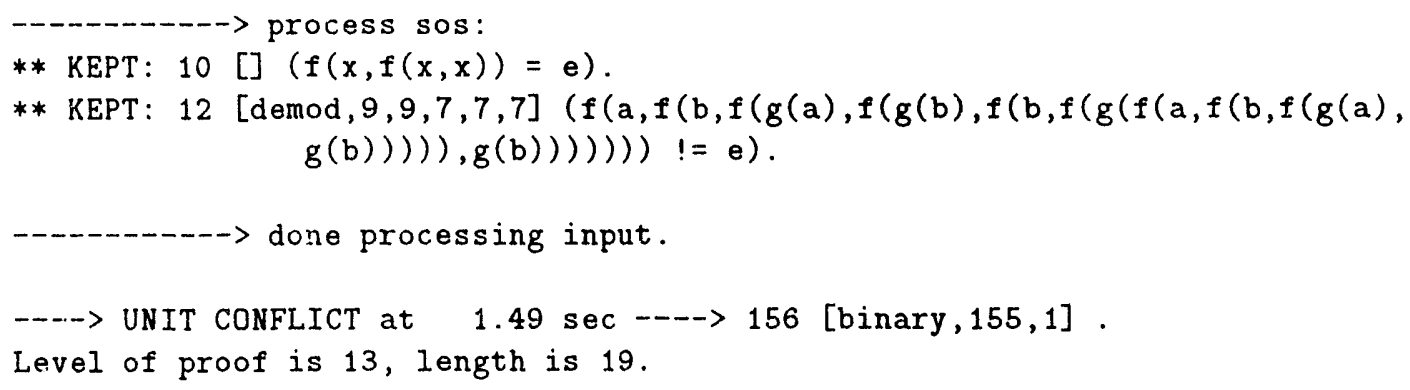




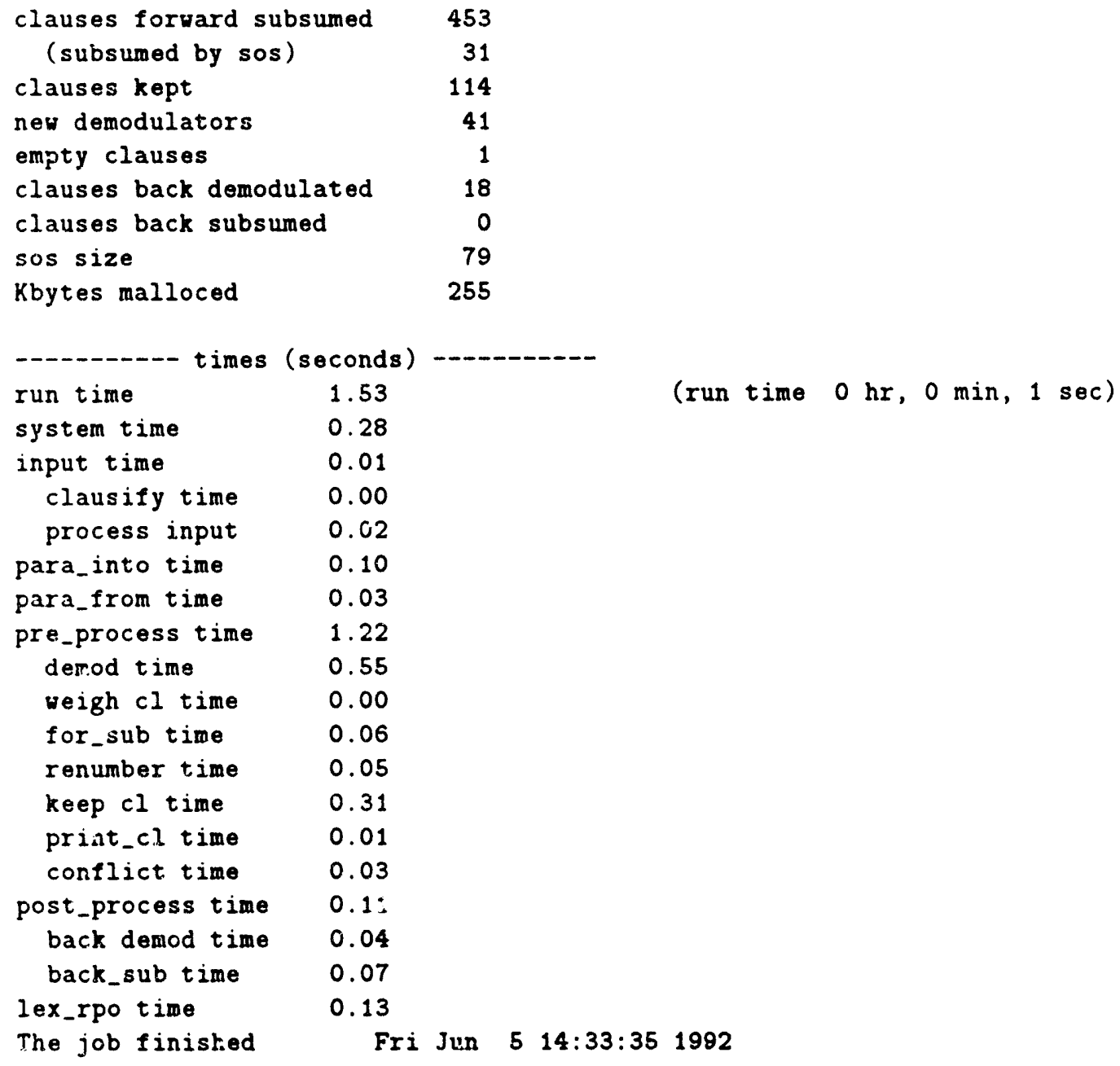




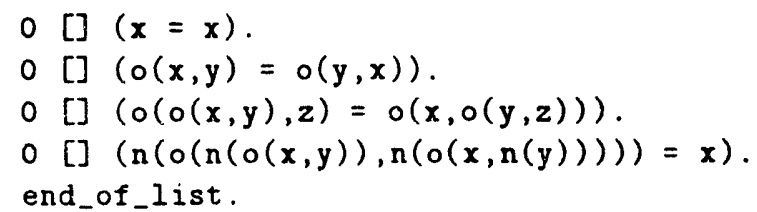

list (sos).

0[]$(o(c, c)=c)$.

0 [] $(o(n(o(a, n(b))), n(o(n(a), n(b)))) !=b)$.

end_of_list.

OTTER sets dynamic_demod_all, because knuth_bendix is set.

OTTER clears para_into_right, because knuth_bendix is set.

OTTER sets back_demod, because knuth_bendix is set.

OTTER sets para_from, because knuth_bendix is set.

OTTER sets para_into, because knuth_bendix is set.

OTTER clears para_from_right, because knuth_bendix is set.

OTTER sets dynamic_demod, because back_demod is set.

OTTER sets order_eq, because dynamic_demod is set.

$------->$ process usable:

* KEPT: 1[]$(x=x)$.

+++ cannot make into demodulator: 1 [] $(x=x)$.

* $\operatorname{KEPT}: 2[](o(x, y)=o(y, x))$.

+++ cannot make into demodulator: 2[]$(o(x, y)=o(y, x))$.

* KEPT: 3[]$(o(o(x, y), z)=o(x, o(y, z)))$.

* KEPT: 5 [] $(n(o(n(o(x, y)), n(o(x, n(y)))))=x)$.

* KEPT: 7 [

** KEPT: 9 [] $(o(n(o(a, n(b))), n(o(n(a), n(b)))) !=b)$.

(n) processing input.

Resetting weight 1 imit to 18.

Resetting weight limit to 17.

-- $>$ UNIT CONFLICT at $98.19 \mathrm{sec}-\ldots 7578$ [binary, 7577,1].

Level of proof is 31 , length is 62 .

1[]$(x=x)$.

2[]$(o(x, y)=o(y, x))$.

$4,3[](o(o(x, y), z)=o(x, o(y, z)))$.

5[]$(n(o(n(o(x, y)), n(o(x, n(y)))))=x)$.

$8,7[](o(c, c)=c)$.

9[]$(o(n(o(a, n(b))), n(o(n(a), n(b)))) !=b)$.

10 [para_from, 7,5$](n(o(n(c), n(o(c, n(c)))))=c)$.

13,12 [para_from, 7, 3] $(o(c, o(c, x))=o(c, x))$.

15,14 [para_into, 12, 2] $(o(c, o(x, c))=o(c, x))$.

16 [para_from, 12,5] $(n(o(n(o(c, x)), n(o(c, n(o(c, x))))))=c)$.

18 [para...into, 14, 3] $(o(c, o(x, o(y, c)))=o(c, o(x, y)))$.

20 [para_from, 14,5] $(\mathrm{n}(o(\mathrm{n}(o(c, x)), \mathrm{n}(o(c, n(o(x, c))))))=c)$.

23,22 [para_from, 14, 3, demod, 4, 4] $(o(c, o(x, o(c, y)))=o(c, o(x, y)))$.

26 [para_into, 10,2] $(\mathrm{n}(\mathrm{o}(\mathrm{n}(\mathrm{o}(\mathrm{c}, \mathrm{n}(\mathrm{c}))), \mathrm{n}(\mathrm{c})))=\mathrm{c})$. 


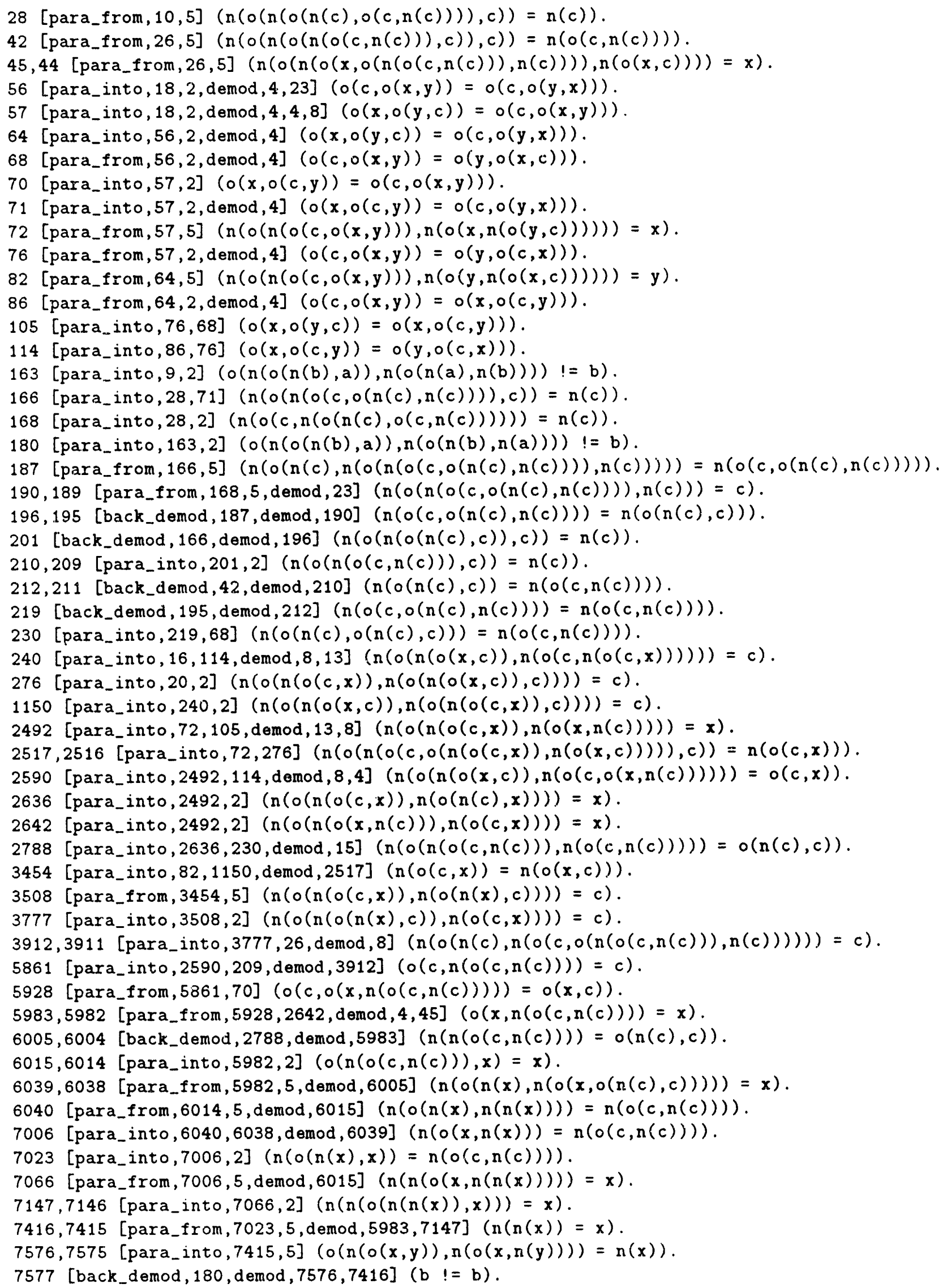




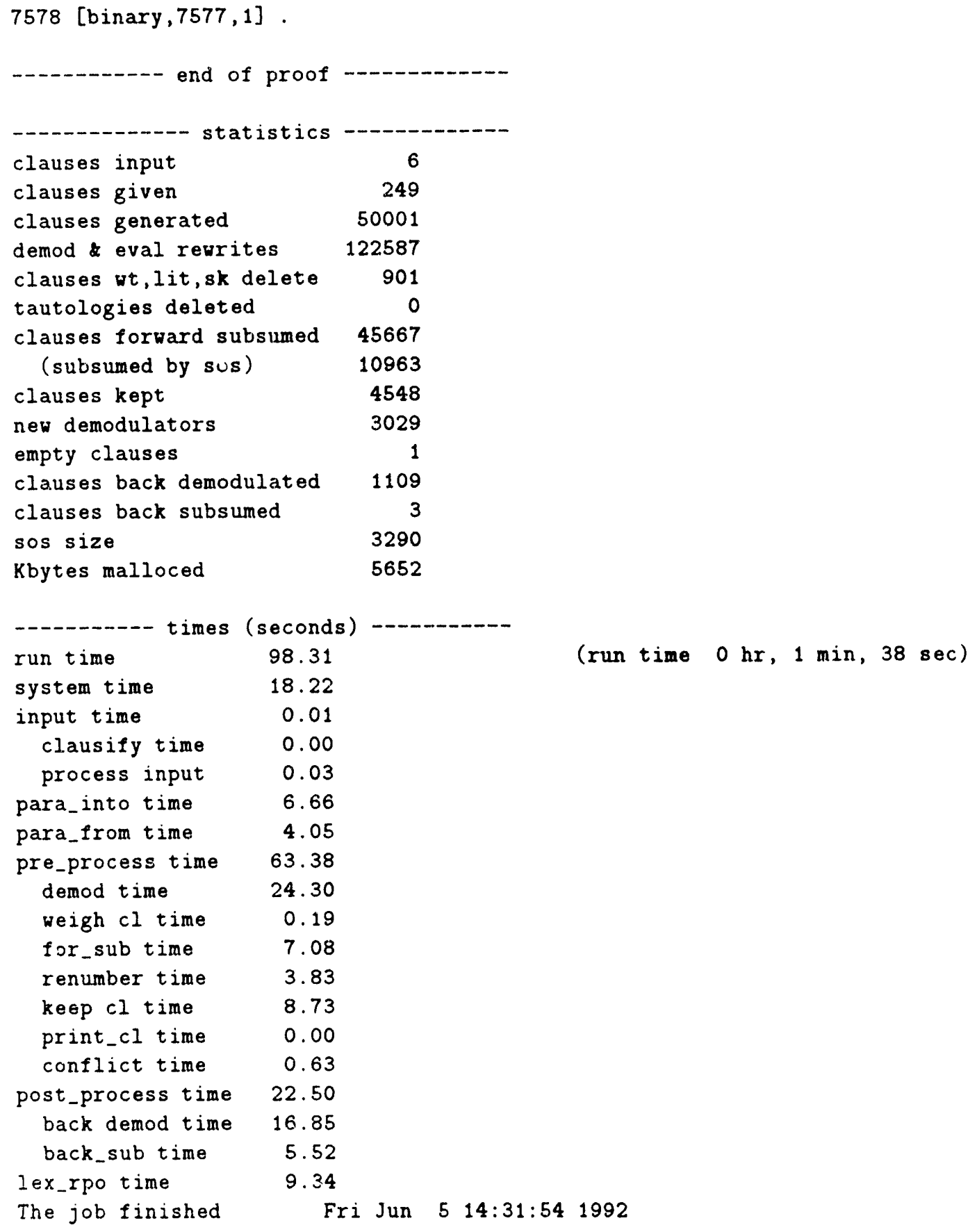

\subsection{Theorem EQ-3: On Ternary Boolean Algebra}

----- OTTER 2.2, July 1991 -.---

The job began on altair.mcs.anl.gov, Fri Jun 5 07:39:41 1992

The command was "otter 22 ".

set (knuth_bendix).

set (index_for_back_demod).

set (process_input).

assign (max_mem, 16000). 


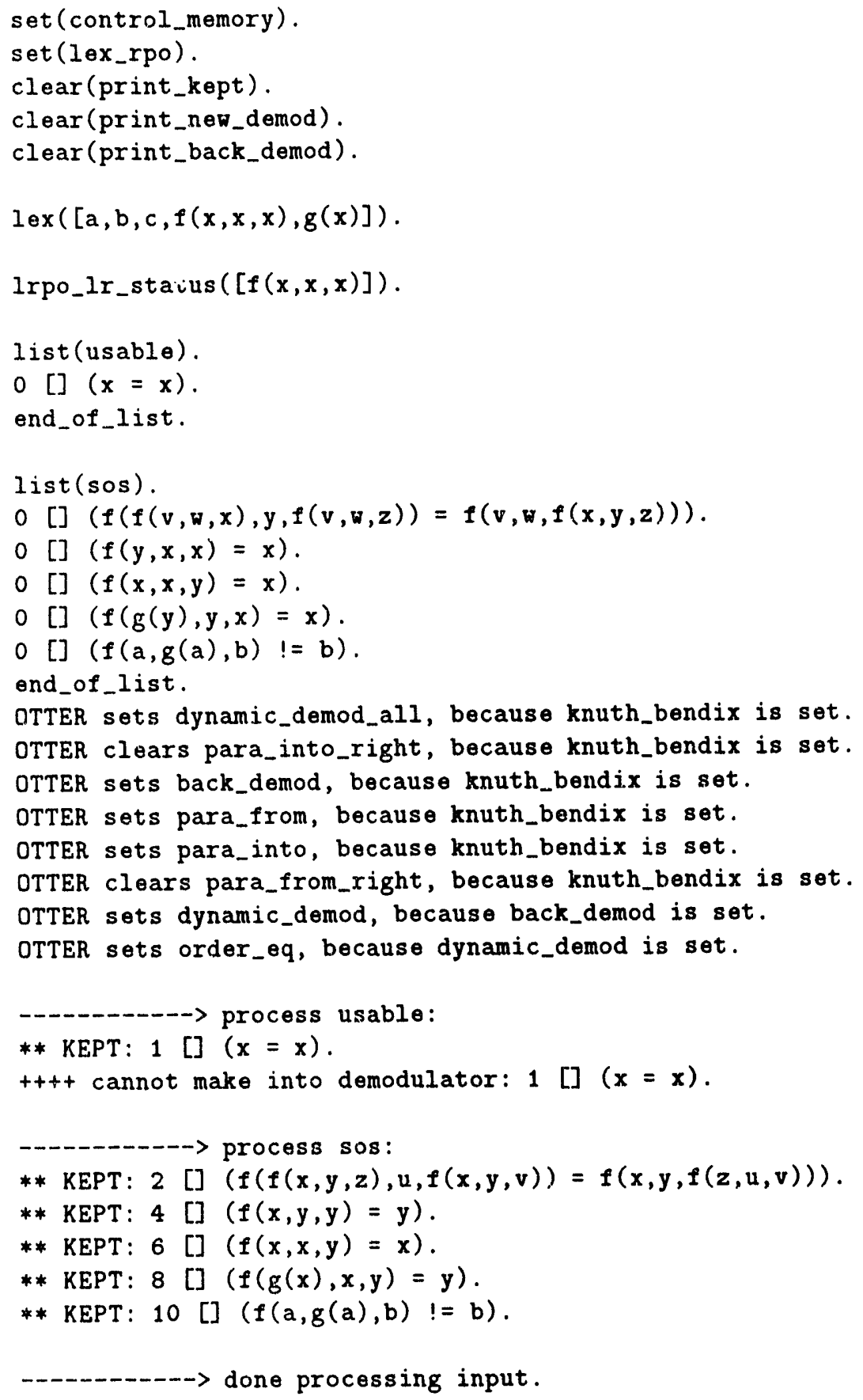

$3,2[](f(f(x, y, z), u, f(x, y, v))=f(x, y, f(z, u, v)))$.

$5,4[](f(x, y, y)=y)$.

$7,6[](f(x, x, y)=x)$.

$9,8[](f(g(x), x, y)=y)$.

10[]$(f(a, g(a), b) !=b)$.

$12,11$ [para_into, 2,6, demod $, 7,7](f(x, y, x)=x)$. 


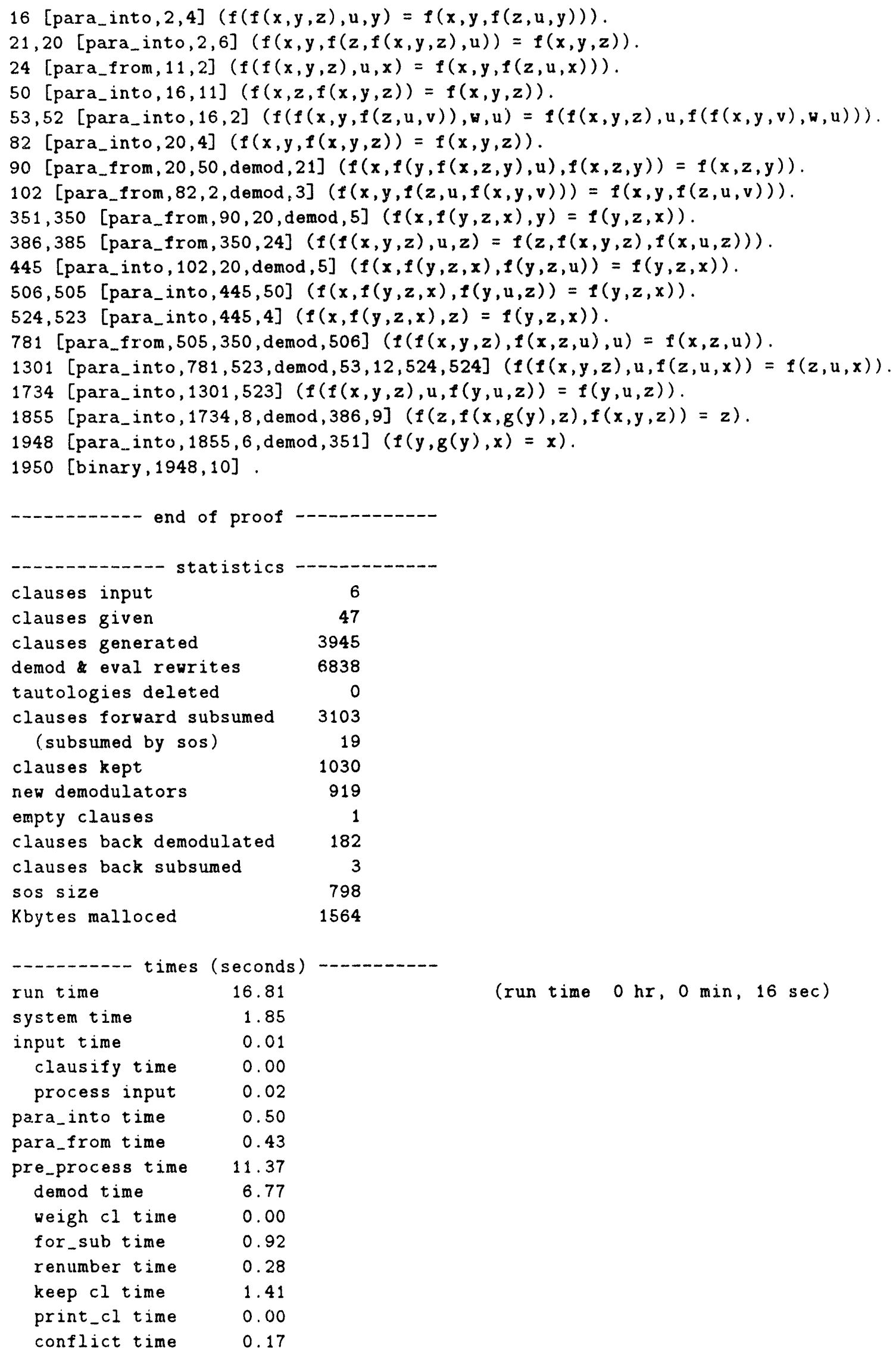




\begin{tabular}{|c|c|}
\hline post_process time & 4.32 \\
\hline back demod time & 3.01 \\
\hline back_sub time & 1.30 \\
\hline lex_rpo time & 0.40 \\
\hline The job finished & Fri Jun \\
\hline
\end{tabular}

\subsection{Theorem EQ-4: Group Theory Single Axiom}

-.-- OTTER 2.2, July 1991 - -

The job began on altair.mcs.anl.gov, Fri Jun 5 07:36:59 1992

The command was "otter 22 ".

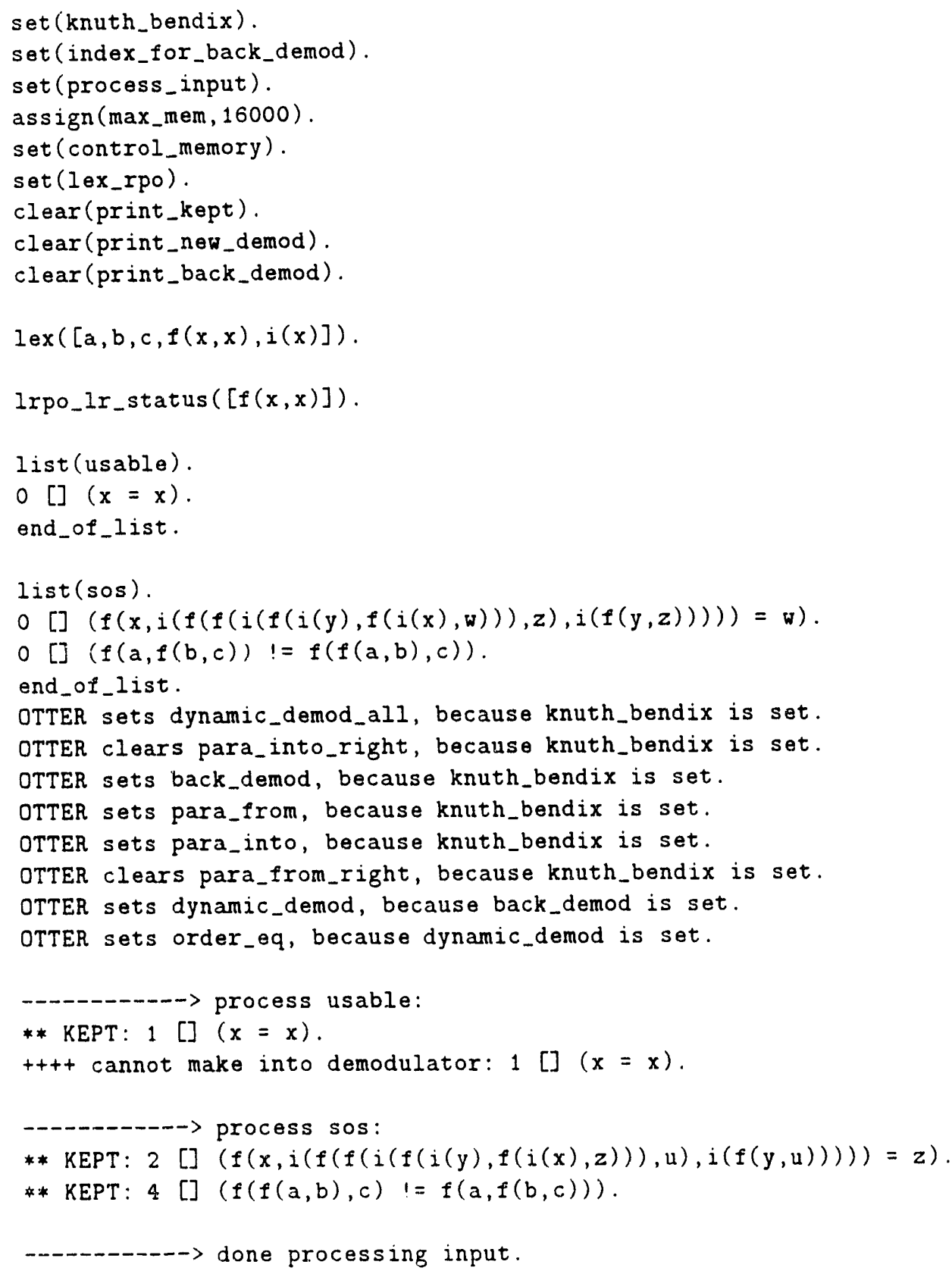


$--->$ UNIT CONFLICT at $44.12 \mathrm{sec}---->4292$ [binary, 4090,4$]$.

Level of proof is 50 , length is 92 .

PROOF

$3,2[](f(x, i(f(f(i(f(i(y), f(i(x), z))), u), i(f(y, u)))))=z)$.

4[]$(f(f(a, b), c) !=f(a, f(b, c)))$.

5 [para_into, 2, 2] $(f(x, i(f(f(i(f(i(y), z)), u), i(f(y, u)))))=i(f(f(i(f(i(v)$, $f(i(i(x)), z))), w), i(f(v, w)))))$.

12 [para_into, 5, 5] $(f(x, i(f(i(f(f(i(f(i(y), f(i(i(i(f(i(z), u)))), v))), w)$, $i(f(y, w)))), i(f(z, i(f(f(i(f(i(v 6), v)), v 7), i(f(v 6, v 7)))))))))=$ $i(f(f(i(f(i(v 8), f(i(i(x)), u))), v 9), i(f(v 8, v 9)))))$.

19,18 [para_into,5,2] $(i(f(f(i(f(i(y), f(i(i(z)), f(i(z), x)))), u), i(f(y, u))))=x)$.

29 [para_into, 18,18, demod,19] $(i(f(f(i(f(i(x), f(i(y), f(y, w)))), v 6), i(f(x, v 6))))=w)$.

48 [para_into, 27,5] $(i(f(i(f(f(i(f(i(x), f(i(i(i(f(i(y), f(i(z), f(z, u)))))), v)))$, w),$i(f(x, w)))), i(f(y, i(f(f(i(f(i(v 6), v)), v 7), i(f(v 6, v 7))))))))=u)$.

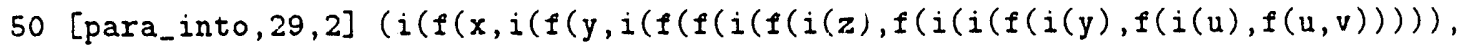
$x))), w), i(f(z, w)))))))=v)$.

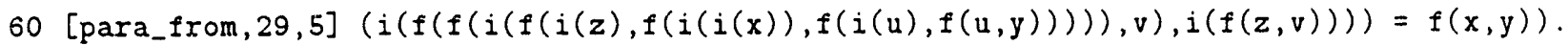

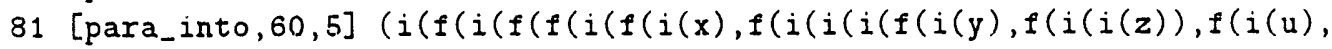
$f(u, v))))))), w))), v 6), i(f(x, v 6)))), i(f(y, i(f(f(i(f(i(v 7), w))$, v8), $i(f(v 7, v 8))))))))=f(z, v))$.

98,97 [para_from, 60,5] $(i(f(f(i(f(i(u), f(i(i(x)), f(i(i(y)), f(i(v), f(v, z))))))$, w), $i(f(u, w))))=f(x, f(y, z)))$.

99 [para_from, 30, 2] ( $f(i(x), f(x, y))=f(i(z), f(z, y)))$.

114 [para_into, 99,99$](f(i(i(x)), f(i(y), f(y, z)))=f(i(u), f(u, f(x, z))))$. 116,115 [para_into, 99,5, demod, 3] $(f(i(v), f(v, i(f(f(i(f(i(w), z)), v 6), i(f(w, v 6))))))=z)$.

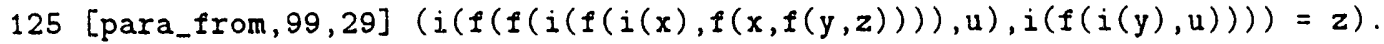

136 [para_from, 99,2] $(f(x, i(f(f(i(f(i(y), f(y, z))), u), i(f(i(x), u)))))=z)$.

146 [para_from, 114,60, demod,98] $(f(y, f(i(z), f(z, v)))=f(y, f(i(v 6), f(v 6, v))))$.

158 [para_into, 146, 99] $(f(x, f(i(i(y)), f(i(z), f(z, u))))=f(x, f(i(v), f(v, f(y, u)))))$.

160 [para_from, 146, 99] $(f(i(x), f(x, f(i(y), f(y, z))))=f(i(u), f(u, f(i(v), f(v, z)))))$.

210 [para_into, 115, 99] ( $f(i(x), f(x, i(f(f(i(f(i(y), f(y, z))), u), i(f(v, u))))))=f(v, z))$.

221,220 [para_into, 115, 99] $(f(i(x), f(x, i(f(f(i(y), f(y, z)), i(f(u, f(f(i(u), v), z)))))))=v)$.

224 [para_into, 115,2] ( $f(i(x), f(x, i(f(y, i(f(z, i(f(f(i) f(i(u), f(i(i(f(i(z), v)))$, y))), w), i(f(u,w)))) )))) )) = v).

300,299 [para_into, 125,99] $(i(f(f(i(f(i(x), f(x, f(y, z)))), f(y, u)), i(f(i(v), f(v, u)))))=z)$. 355 [para_into, 136, 99] $(f(x, i(f(f(i(y), f(y, z)), i(f(i(x), f(f(i(u), f(u, v)), z))))))=v)$.

1301 [para_into, 355, 220, demod, 221] $(f(f(i(x), f(x, y)), i(f(v 6, i(v 6))))=y)$.

1410 [para_from, 1301,210] $(f(i(f(i(x), f(x, y))), y)=f(i(f(i(z), f(z, u))), u))$.

1434 [para_from, 1301,220] $(f(i(x), f(x, i(f(f(i) f(i(y), f(y, z))), z), i(f(u$, $f(f(i(u), v), i(f(w, i(w))))))))))=v)$.

1436 [para_from, 1301,114] $(f(i(i(x)), f(i(f(i(y), f(y, z))), z))=$ $f(i(u), f(u, f(x, i(f(v, i(v)))))))$.

1484 [para_from, 1410,355] $(f(f(i(x), f(x, f(f(i(y), f(y, z)), u))), i(f(f(i(v))$ $f(v, u)), i(f(i(f(i(u), f(w, v 6))), v 6)))))=z)$.

1489,1488 [para_from, 1410,220, demod, 221] $(f(i(i(v)), f(i(f(i(w), f(w, v 6))), v 6))=v)$.

1493,1492 [para_from, 1410,210] $(f(i(x), f(x, i(f(f(i(f(i(y), f(y, z))), z), i(f(u, v))))))=f(u, v))$.

1541 [back_demod, 1436, demod, 1489] $(f(i(u), f(u, f(x, i(f(v, i(v))))))=x)$.

1545,1544 [back_demod, 1434, demod, 1493] $(f(u, f(f(i(u), v), i(f(w, i(w)))))=v)$.

1591 [para_from, 1541,1301] $(f(x, i(f(y, i(y))))=f(x, i(f(z, i(z)))))$.

1675 [para_into, 1544,5] $(f(x, f(i(f(f(i(f(i(y), f(i(i(i(x))), z))), u), i(f(y, u))))$, $i(f(v, i(v)))))=i(f(f(i(f(i(w), z)), v 6), i(f(w, v 6)))))$.

1802 [para_from, 1488, 210] $(f(i(x), f(x, i(f(f(i) f(i(y), f(y, z))), f(i(f(i(u)$, 
$f(u, v))), v)), i(w)))))=f(i(i(w)), z))$.

1857 [para_from, 1591,1488] $(f(i(i(x)), f(i(f(i(y), f(y, i(f(z, i(z)))))), i(f(u, i(u)))))=x)$.

1884 [para_from, 1591,115, demod, 116] $(i(f(z, i(z)))=i(f(v, i(v))))$.

1948 [para_from, 1884,1488, demod,1489] $(f(x, i(x))=f(u, i(u)))$.

1969 [para_from, 1884, 99] $(f(i(f(x, i(x))), f(f(y, i(y)), z))=f(i(u), f(u, z)))$.

2010 [para_from, 1948,1544] $(f(x, f(y, i(y)))=i(i(x)))$.

2107 [para_into, 2010,99] $(f(i(x), f(x, i(y)))=i(i(i(y))))$.

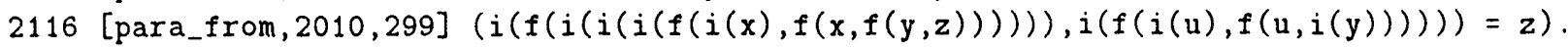

2131 [para_from, 2010,210] $(f(i(x), f(x, i(f(i(i(i(f(i(y), f(y, z))))), i(f(u, f(v, i(v))))))))=f(u, z)$

2368 [para_into, 2107, 299, demod, 300] $(f(i(x), f(x, y))=i(i(y)))$.

2373 [para_into, 2368, 1884] $(f(i(f(x, i(x))), f(f(y, i(y)), z))=i(i(z)))$.

2381,2380 [para_into, 2368,2368] $(i(i(f(x, y)))=f(i(i(x)), i(i(y))))$.

2401 [para_into, 2368,158, demod, $2381,2381,2381](f(i(x), f(x, f(i(y), f(y, f(z, u)))))=$ $f(i(i(i(i(z)))), f(i(i(i(v))), f(i(i(v)), i(i(u))))))$.

2438 [back_demod, 2131, demod, 2381,2381] ( $f(i(x), f(x, i(f(i(f(i) i(i(y))), f(i(i(y))$, $i(i(z))))), i(f(u, f(v, i(v))))))))=f(u, z))$.

2446 [back_demod, 2116, demod, 2381,2381,2381] (i(f(i) $(i(i(i(x))), f(i(i(x)), f(i(i(y))$, $i(i(z)))))), i(f(i(u), f(u, i(y))))))=z)$.

2587 [back_demod, 224, demod, 2381] ( $f(i(x), f(x, i(f(y, i(f(z, i(f(f(i) f(i(u), f(f(i(i) i(z)))$, $i(i(v))), y))), w), i(f(u, w)))))))))=v)$.

2598 [back_demod, 81, demod, 2381, 2381, 2381,2381] ( $i(f(i) f(f(i) f(i(x), f(i(f(i) i(i(y)))$, $f(i(i(i(i(z)))), f(i(i(i(u))), f(i(i(u)), i(i(v))))))), w))), v 6), i(f(x, v 6))))$, $i(f(y, i(f(f(i(f(i(v 7), w)), v 8), i(f(v 7, v 8))))))))=f(z, v))$.

2604 [back_demod, 50, demod, $2381,2381,2381]$ ( $i(f(x, i(f(y, i) f(f(i) f(i(z), f(f(i) i(i(y)))$, $f(i(i(i(u))), f(i(i(u)), i(i(v))))), x))), w), i(f(z, w)))))))=v)$.

2606 [back_demod, 48, demod, 2381,2381,2381] ( $(f(i) f(f(i) f(i(x), f(i(f(i) i(i(y)))$, $f(i(i(i(z))), f(i(i(z)), i(i(u)))))), v))), w), i(f(x, w)))), i(f(y$, $i(f(f(i(f(i(v 6), v)), v 7), i(f(v 6, v 7))))))))=u)$.

2617 [back_demod, 12, demod, 2381] $(f(x, i(f(i(f(f(i(f(i(y), f(i(f(i(i(i(z))), i(i(u))))$, $v))), w), i(f(y, w)))), i(f(z, i(f(f(i(f(i(v 6), v)), v 7), i(f(v 6, v 7)))))))))=$ $i(f(f(i(f(i(v 8), f(i(i(x)), u))), v 9), i(f(v 8, v 9)))))$.

2621 [para_from, 2368,1488$](i(i(x))=f(i(y), f(y, x)))$.

2633 [para_from, 2368,210] $(f(i(x), f(x, i(f(i(i(y)), i(f(z, f(f(i(u), f(u, v)), y)))))))=f(z, v))$.

2647 [para_from, 2368,1301] $(f(i(i(x)), i(f(y, i(y))))=x)$.

2706 [para_from, 2368,160] $(f(i(x), f(x, f(i(i(y)), i(i(z)))))=f(i(u), f(u, f(i(v), f(v, f(y, z))))))$.

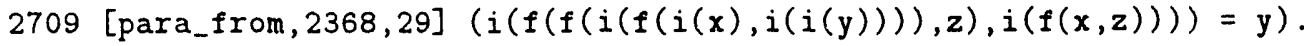

2802,2801 [para_from, 2621,1488] $(f(i(f(i(x), f(x, y))), f(i(f(i(z), f(z, u))), u))=i(y))$.

2866, 2865 [back_demod, 1802, demod, 2802] $(f(i(x), f(x, i(f(i(z), i(w)))))=f(i(i(w)), z))$.

2873 [back_demod, 2633, demod, 2866,2381,2381,2381,2381] $(f(f(i(i(z)), f(f(i(i(i(u)))$, $f(i(i(u)), i(i(v)))), i(i(y)))), i(y))=f(z, v))$.

2879 [back_demod, 2438, demod, 2866,2381,2381] $(f(f(i(i(u)), f(i(i(v)), i(i(i(v)))))$, $f(i(i(i(y))), f(i(i(y)), i(i(z)))))=f(u, z))$.

2884,2883 [para_into, 2647,2621] $(f(i(f(i(x), f(x, y))), i(f(z, i(z))))=i(y))$.

2921,2920 [back_demod, 1857, demod, 2884, 2381] $(f(i(i(x)), f(i(i(z)), i(i(i(z)))))=x)$.

2929,2928 [back_demod, 2879, demod, 2921] $(f(x, f(i(i(i(z))), f(i(i(z)), i(i(u)))))=f(x, u))$.

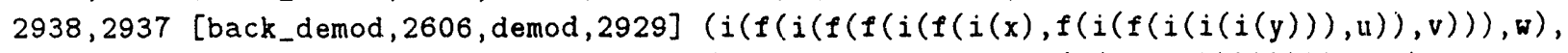
$i(f(x, v)))), i(f(y, i(f(f(i(f(i(v 6), v)), v 7), i(f(v 6, v 7))))))))=u)$.

2940,2939 [back_demod, 2604, demod, 2929] ( $i(f(x, i(f(y, i) f(f(i) f(i(z), f(f(i) i(i(y)))$, v),$x))), w), i(f(z, w))))))))=v)$.

2944,2943 [back_demod, 2598, demod, 2929,2938] $(f(i(i(i(i(z)))), v)=f(z, v))$.

2978,2977 [back_demod, 2401, demod, 2929, 2944] $(f(i(x), f(x, f(i(y), f(y, f(z, u)))))=f(z, u))$.

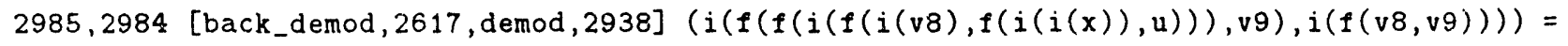
$f(x, i(i(u))))$.

2987,2986 [back_demod, 2587, demod, 2940] $(f(i(x), f(x, i(i(v))))=v)$. 


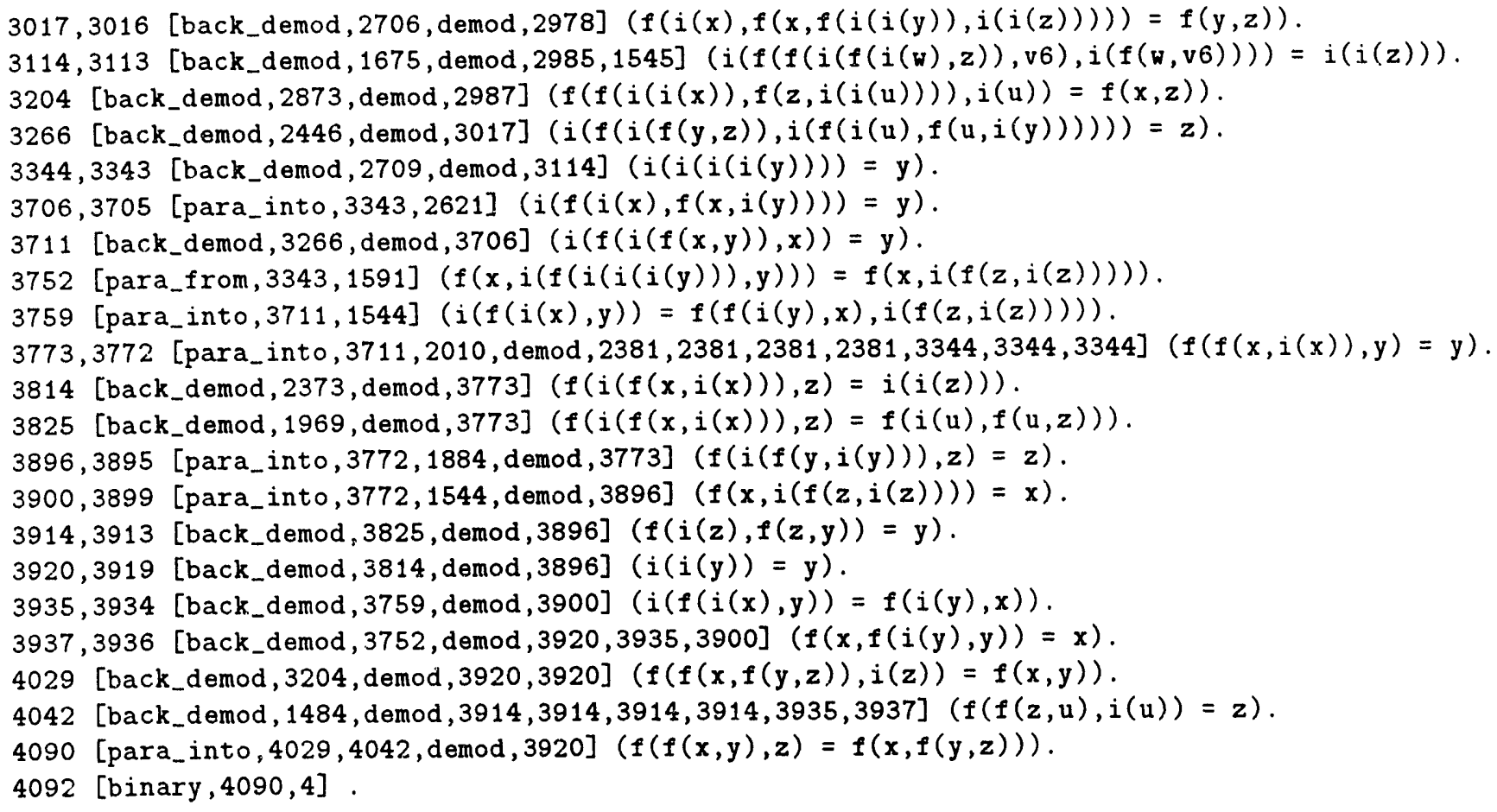

\begin{tabular}{|c|c|c|}
\hline \multicolumn{2}{|l|}{ clauses input } & 3 \\
\hline \multicolumn{2}{|l|}{ clauses given } & 57 \\
\hline \multicolumn{2}{|l|}{ clauses generated } & 3417 \\
\hline \multicolumn{2}{|c|}{ demod \& eval rewrites } & 9814 \\
\hline \multicolumn{2}{|c|}{ tautologies deleted } & 0 \\
\hline \multirow{2}{*}{\multicolumn{2}{|c|}{$\begin{array}{l}\text { clauses forward subsumed } \\
\text { (subsumed by sos) }\end{array}$}} & 3327 \\
\hline & & 503 \\
\hline \multicolumn{2}{|l|}{ clauses kept } & 2507 \\
\hline \multicolumn{2}{|l|}{ new demodulators } & 1584 \\
\hline \multicolumn{2}{|l|}{ empty clauses } & 1 \\
\hline \multicolumn{2}{|c|}{ clauses back demodulated } & 2414 \\
\hline \multicolumn{2}{|c|}{ clauses back subsumed } & 61 \\
\hline \multicolumn{2}{|l|}{ sos size } & 15 \\
\hline \multicolumn{2}{|l|}{ Kbytes malloced } & 4470 \\
\hline$\ldots \ldots$ times & (seconds) & \\
\hline run time & 89.93 & \\
\hline system time & 3.46 & \\
\hline input time & 0.01 & \\
\hline clausify time & 0.00 & \\
\hline process input & 0.01 & \\
\hline para_into time & 0.69 & \\
\hline para_from time & 0.83 & \\
\hline pre_process time & 71.63 & \\
\hline demod time & 7.86 & \\
\hline weigh cl time & 0.00 & \\
\hline for_sub time & 1.73 & \\
\hline renumber time & 0.84 & \\
\hline
\end{tabular}




$\begin{array}{cc}\text { keep cl time } & 9.14 \\ \text { print_cl time } & 0.00 \\ \text { conflict time } & 0.32 \\ \text { post_process time } & 16.45 \\ \text { back demod time } & 14.77 \\ \text { back_sub time } & 1.43 \\ \text { lex_rpo time } & 2.54 \\ \text { The job finished } & \text { Fri Jun } 507: 38: 341992\end{array}$

\subsection{Theorem EQ-5: On Wajsberg Algebra}

--- OTTER 2.2, July 1991 -

The job began on altair.mcs.anl.gov, Thu Jun 4 17:31:43 1992

The command was "otter 22 ".

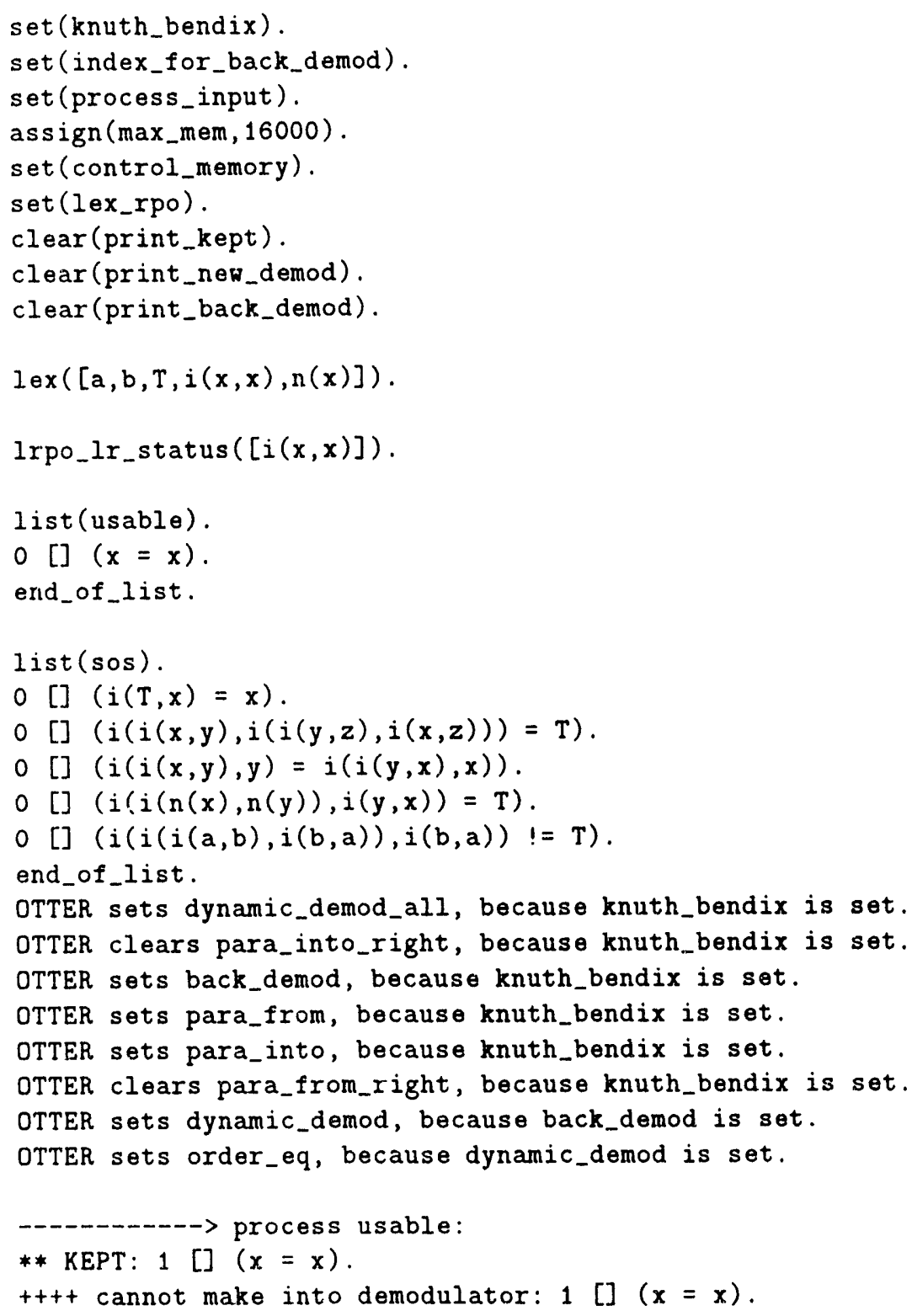


$\rightarrow$ process sos

** KEPT: 2 [] $(i(T, x)=x)$.

** KEPT: 4[]$(i(i(x, y), i(i(y, z), i(x, z)))=T)$.

** KEPT: 6 [] $(i(i(x, y), y)=i(i(y, x), x))$.

++++ cannot make into demodulator: 6[]$(i(i(x, y), y)=i(i(y, x), x))$.

** KEPT: 7 [] $(i(i(n(x), n(y)), i(y, x))=T)$.

** KEPT: 9 [] $(i(i(i(a, b), i(b, a)), i(b, a)) !=T)$.

-...-. done processing input.

Resetting weight limit to 15 .

$--->$ UNIT CONFLICT at $2248.86 \mathrm{sec}---\rightarrow 11462$ [binary, 11460,11350 ] .

Level of proof is 37 , length is 85 .

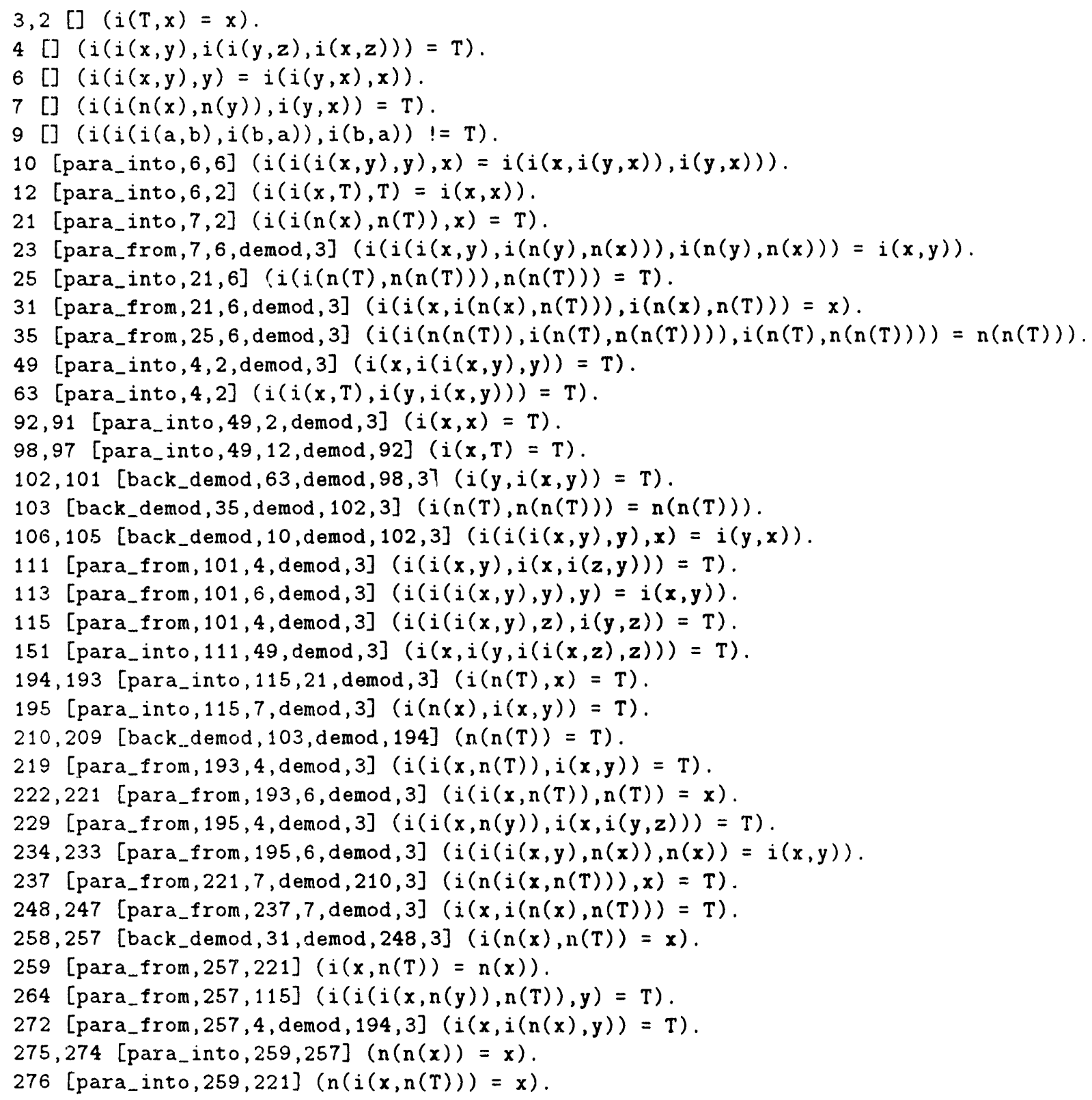


278 [para_from, 259,113, demod, 222] $(n(x)=i(x, n(T)))$.

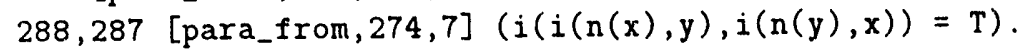

289 [para_from, 274,7] $(i(i(x, n(y)), i(y, n(x)))=T)$.

291 [para_from, 278,7] $(i(i(n(x), i(y, n(T))), i(y, x))=T)$.

322,321 [para_from, 219,6 , demod, 3] $(i(i(i(x, y), i(x, n(T))), i(x, n(T)))=i(x, y))$.

377 [para_into, 151,6] $(i(x, i(i(i(i(x, y), y), z), z))=T)$.

417 [para_into, 264,259] $(i(n(i(x, n(y))), y)=T)$.

429 [para_into, 417,274] $(i(n(i(x, y)), n(y))=T)$.

441 [para_into, 429,6] $(i(n(i(i(x, y), y)), n(x))=T)$.

474,473 [para_into, 287,274] $(i(i(x, y), i(n(y), n(x)))=T)$.

479 [para_into, 287, 272, demod, 275, 3] $(i(n(i(x, y)), x)=T)$.

491 [back_demod, 23, demod, 474,3] $(i(n(y), n(x))=i(x, y))$.

496 [para_from, 287,6, demod, 3, 288,3] $(i(n(x), y)=i(n(y), x))$.

500,499 [para_into, 479, 278] $(i(i(i(x, y), n(T)), x)=T)$.

509 [para_into, 491,278] $(i(i(x, n(T)), n(y))=i(y, x))$.

511 [para_into, 491,274] $(i(x, n(y))=i(y, n(x)))$.

513 [para_into, 491,276] $(i(n(x), y)=i(i(y, n(T)), x))$.

520 [para_from, 491,4] $(i(i(n(x), y), i(i(y, n(z)), i(z, x)))=T)$.

530 [para_from, 491,113, demod, 234] $(i(x, y)=i(n(y), n(x)))$.

533 [para_from, 491,49] $(i(n(x), i(i(y, x), n(y)))=T)$.

536,535 [para_from, 491,6] $(i(i(n(x), n(y)), n(y))=i(i(x, y), n(x)))$.

540 [para_into, 496,276] $(i(x, y)=i(n(y), i(x, n(T))))$.

565 [para_from, 496,6] $(i(i(n(x), y), x)=i(i(x, n(y)), n(y)))$.

569 [para_into, 511, 276] $(i(x, y)=i(i(y, n(T)), n(x)))$.

598 [para_from, 511,6] $(i(i(x, n(y)), n(x))=i(i(n(x), y), y))$.

624,623 [para_into, 530,105] $(i(n(y), n(i(i(y, x), x)))=i(x, y))$.

627 [para_into, 530,6] $(i(n(x), n(i(y, x)))=i(i(x, y), y))$.

647 [para_from,530,4] $(i(i(x, y), i(i(n(z), n(y)), i(x, z)))=T)$.

723 [para_into, 289, 278] $(i(i(x, i(y, n(T))), i(y, n(x)))=T)$.

726,725 [para_into, 289,530] $(i(n(i(x, n(y))), n(i(y, n(x))))=T)$.

771 [para_from, 441,6, demod, 3,624] $(i(i(y, x), n(i(i(x, y), y)))=n(x))$.

808 [para_into, 509,276] $(i(i(x, n(T)), y)=i(i(y, n(T)), x))$.

1005 [para_from, 533,4, demod, 3] ( $i(i(i(i(x, y), n(x)), z), i(n(y), z))=T)$.

1464,1463 [para_into, 229,569, demod, 258] $(i(i(x, n(y)), i(y, i(x, z)))=T)$.

$1470,1469$ [para_into, 229,513, demod, 258] ( $i(i(x, y), i(n(y), i(x, z)))=T)$.

2855 [para_into, 9,530$](i(i(i(n(b), n(a)), i(b, a)), i(b, a)) !=T)$.

7827,7826 [para_from, 725,627, demod, 275,726,3] $(n(i(y, n(x)))=i(i(x, n(y)), n(T)))$.

8405,8404 [para_into, 771,723, demod, 1464,3,3,7827] $(n(i(y, i(x, n(T))))=i(i(y, n(x)), n(T)))$.

8431,8430 [para_into, 771,291 , demod, 1470,3,8405,3] $(n(i(x, y))=i(i(n(y), n(x)), n(T)))$.

10857 [para_from, 565,113, demod, 106] $(i(i(n(y), x), x)=i(i(n(x), y), x))$.

10860 [para_from, 10857,233, demod, 8431,275,8431,275,322] $(i(i(n(x), y), x)=i(i(n(y), x), x))$.

10862 [para_into, 10860, 274, demod,536] $(i(i(x, y), n(x))=i(i(y, x), n(y)))$.

10887 [para_into, 10862,496, demod, 275] $(i(i(n(x), y), y)=i(i(x, n(y)), n(x)))$.

10936 [para_from, 10887, 598, demod, $275,8431,275,275,8431,275,275,500,3]$

$$
(i(i(i(x, y), n(x)), i(i(y, x), n(T)))=y) \text {. }
$$

11090 [para_into, 520,540, demod, 258] $(i(i(n(x), y), i(i(x, n(z)), i(z, y)))=T)$.

11114 [para_into, 1005,647, demod, 275, 3] $(i(n(x), i(i(n(y), z), i(i(z, x), y)))=T)$.

11184 [para_into, 11090,808, demod, 8431,275,3,258,258] $(i(i(x, y), i(i(z, x), i(z, y)))=T)$.

11202 [para_from, 11184,377, demod, 3$](i(x, i(i(y, i(x, z)), i(y, z)))=T)$.

$11213,11212$ [para_into, 11202,11202 , demod, 3$](i(i(x, i(y, z)), i(y, i(x, z)))=T)$.

11285 [para_from, 11212,6, demod, $3,11213,3](i(x, i(y, z))=i(y, i(x, z)))$.

11350 [para_from, 11285,2855$](i(b, i(i(i(n(b), n(a)), i(b, a)), a)) !=T)$.

11460 [para_into, 11114,10936 , demod, $275,8431,275,8431,3,222]$

$(i(x, i(i(i(n(x), n(y)), i(x, y)), y))=T)$. 


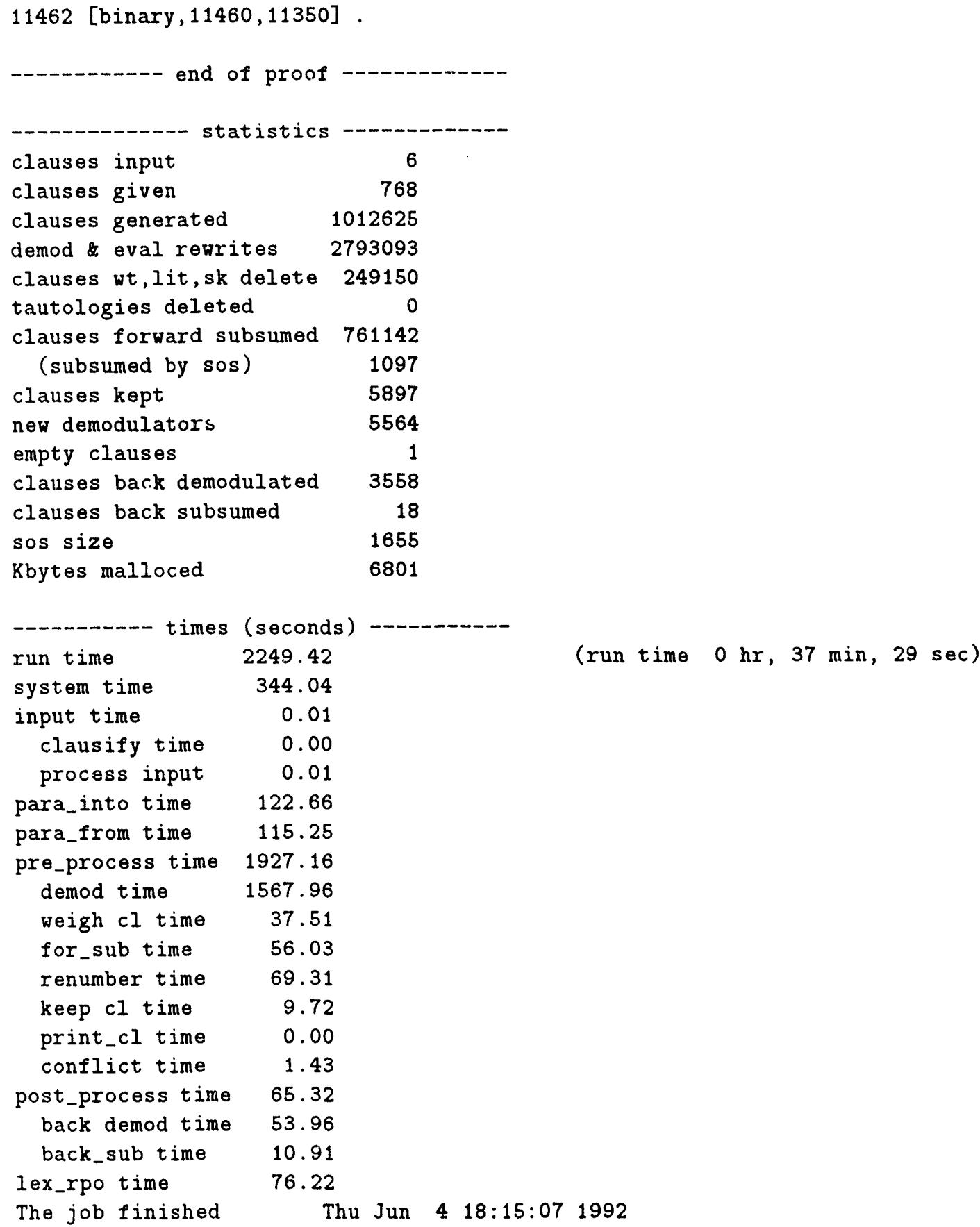

\section{Conclusion}

Years of experimentation with theorem-proving systems $[4,9,3,5,6]$ have enabled us to accumulate a wide variety of variations and parameters to control our basic theorem-proving algorithm. The typical user need know about only a few of them. This contest forced us to consider how we would set them if there were to be a parameterless version of O) $1 \mathrm{ER}$.

Most of the above settings just represent common sense. The value of max mem did have to be carefully chosen so that we could get proofs of all of these theorems with the same. 
value.

We thank Ross Overbeek for proposing this exercise, and we hope that others found it as usf ful as we did.

\section{References}

[1] E. Lusk and IV. Mccune. Experiments with RoO, a parallel automated deduction stistrm. In B. Fronhöfer and G. Wrightson. editors, Parallelization in Infrence Systoms. Lerture lotes in Artificial Intclligoner. Vol. 590, pages 139 162. New York, 1992. Springer-Verlag.

[2] E. Lusk. W. Mc('une. and J. Slaney. Roo-a parallel theorem prover. Tech. Memo Mo'sT.11-19. Mathematics and ('omputer Science Division, Argonme National Laboratory. Argomme, Ill. 1991.

[3] F. L.usk and R. Overbeek. The automated reasoning system ITP. Tech. Report ANLQ1/27. Argonne National Laboratory. Argonne, Ill.. April 19K.4.

[1] J. Mccharen. R. Overbeek, and L. Wos. Problems and experiments for and with automated theorem-proving programs. IEEE Transactions on (ompute rs. ( $2-25(8): 773-7 \times 2$. August 1976 .

[5] W. Mccune. OTter 2.0 Users Giude. Tech. Report ANL-90/9, Argonne National I.ahoratory, Argonne. III., March 1990.

[6] W. Mor'une. What's New in OTter 2.2. Tech. Memo ANL/M('S-TM-153. Mathematirs and Computer Science Division, Argonne National Laboratory, Argonne, Ill., July 1991.

[i] W. Mcc 'une and L. Wos. The absence and the presence of fixed point combinators. The on tical comptuter Sicience. 87:221-228, 1991.

[s] W. Mcc 'une and L. Wos. Experiments in automated deduction with condensed delachment. In D. hapur, editor, Proccedings of the I/th International Confercuce on Automated Deduction. Lecture Notes in Artificial Intelligence, Vol. 607, pages 209 22:3. Vew York. June 1992. Springer-Verlag.

[9] B. Smith. Refremes mahial for the environmental theorem prover: An incarnation of AlRA. Tech. Report ANL-88-2, Argonne National La'soratory, Argonne, Ill., March loxx. 

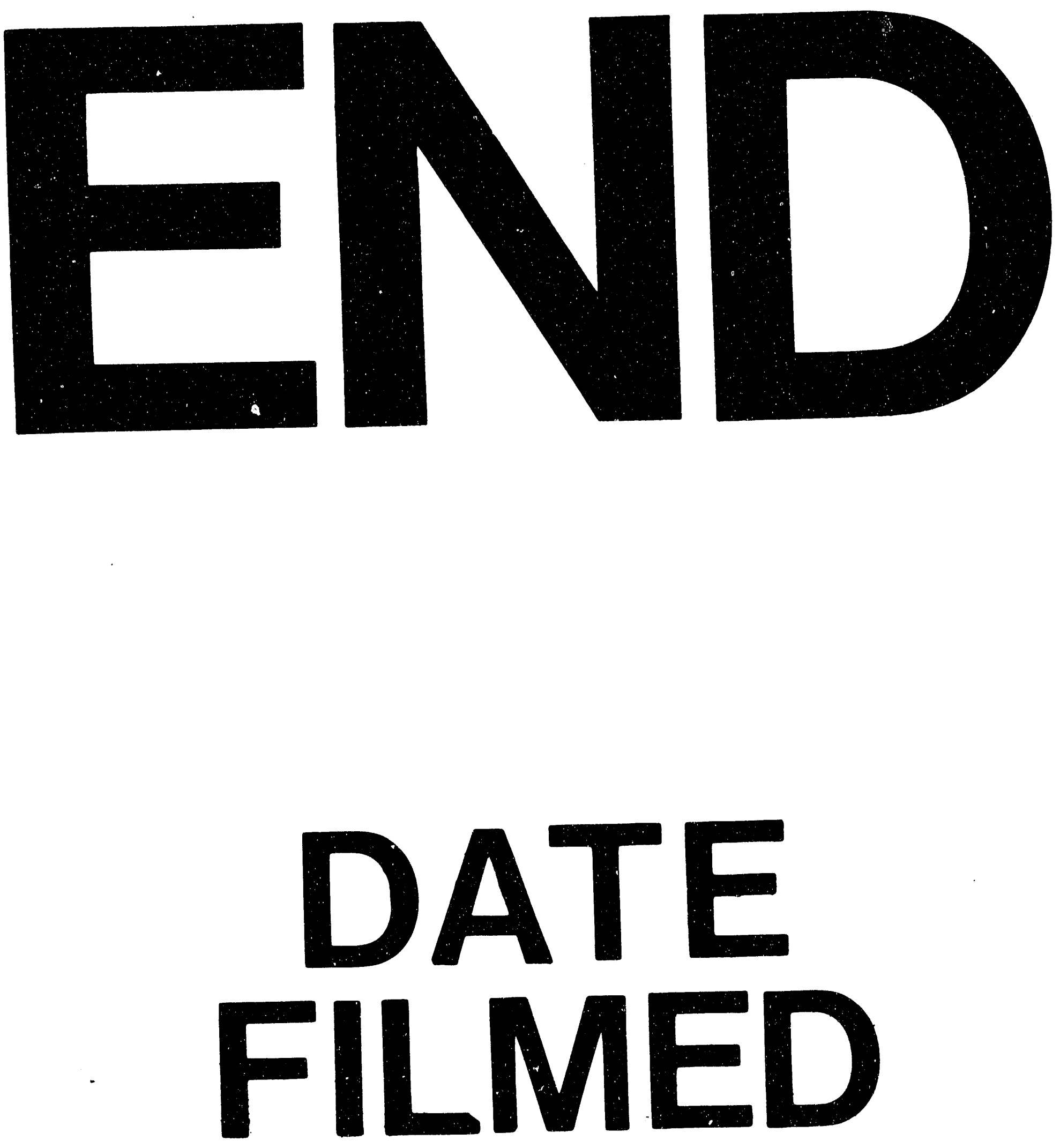

1

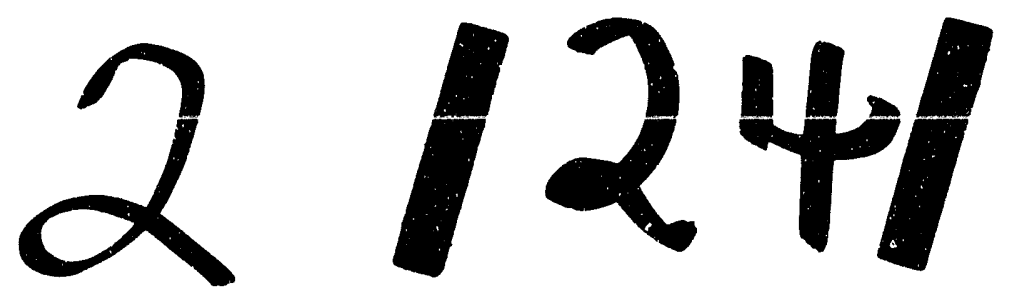

93 
